\title{
ARTICLE
}

Cite this: DOI: 10.1039/x0xx00000x

\section{2-Arylacetic Anhydrides As Ammonium Enolate Precursors}

\author{
Louis C. Morrill, Lyndsay A. Ledingham, Jean-Philippe Couturier, Jasmine Bickel, \\ Andrew D. Harper, Charlene Fallan and Andrew D. Smith*
}

Received 00th January 2012,

Accepted 00th January 2012

DOI: $10.1039 / \mathrm{x} 0 \times x \times 0000 \mathrm{x}$

Readily prepared 2-arylacetic anhydrides act as convenient ammonium enolate precursors in isothiourea (HBTM-2.1)-mediated catalytic asymmetric intermolecular Michael addition-lactonisation processes, giving diverse synthetic building blocks in good yield with high diastereo- and enantiocontrol (up to 95:5

www.rsc.org/ dr and $>99 \%$ ee)

\section{Introduction}

Within the arena of Lewis base catalysis, ${ }^{1}$ the generation and application of ammonium enolates ${ }^{2}$ toward generating stereodefined molecules with high levels of stereocontrol is of widespread interest in asymmetric catalysis. ${ }^{3}$ Most commonly formed from the interaction of a nucleophilic tertiary amine with either a preformed or in situ generated ketene, ${ }^{4} \mathrm{C} 1$ ammonium enolates present an attractive alternative to existing organocatalytic strategies for the generation of enolates or their equivalents such as the use of enamines, ${ }^{5} \mathrm{~N}$-heterocyclic carbenes (NHCs), ${ }^{6}$ and cinchona alkaloid derivatives. ${ }^{7}$

Building upon the pioneering intramolecular nucleophile catalysed aldol-lactonisation (NCAL) strategy developed by Romo, ${ }^{8}$ we have recently shown that isothioureas, ${ }^{9}$ initially employed by Birman and Okamoto as efficient $O$-acyl transfer reagents, ${ }^{10}$ can generate ammonium enolates from carboxylic acids through in situ formation of a mixed anhydride and subsequently undergo a range of intra- and intermolecular Michael addition-cyclisation processes. ${ }^{11}$ While this powerful synthetic strategy allows access to a range of stereodefined products in high enantioselectivity, noteworthy drawbacks include the use of excess sacrificial base (up to 4 equivalents of $i$ - $\operatorname{Pr}_{2} \mathrm{NEt}$ ) and the production of unwanted by-products derived from the acid "activating agent" (such as pivalic anhydride derived from pivaloyl chloride) that can be difficult to separate from the desired products (Figure 1).

As part of our on-going interest in Lewis base catalysis ${ }^{12}$ we wished to investigate alternative bench stable precursors to ammonium enolates at the carboxylic acid oxidation level. Following our recent report on the generation of $\alpha, \beta$ unsaturated acyl ammonium species from homoanhydrides, ${ }^{13}$ and inspired by Connon's functionalisation of enolisable anhydrides using bifunctional squaramides, ${ }^{14}$ in addition to Chi's use of $p$-nitrophenyl esters as azolium enolate precursors, ${ }^{15}$ we envisaged readily available 2-arylacetic anhydrides as C1-ammonium enolate precursors (Figure 2).

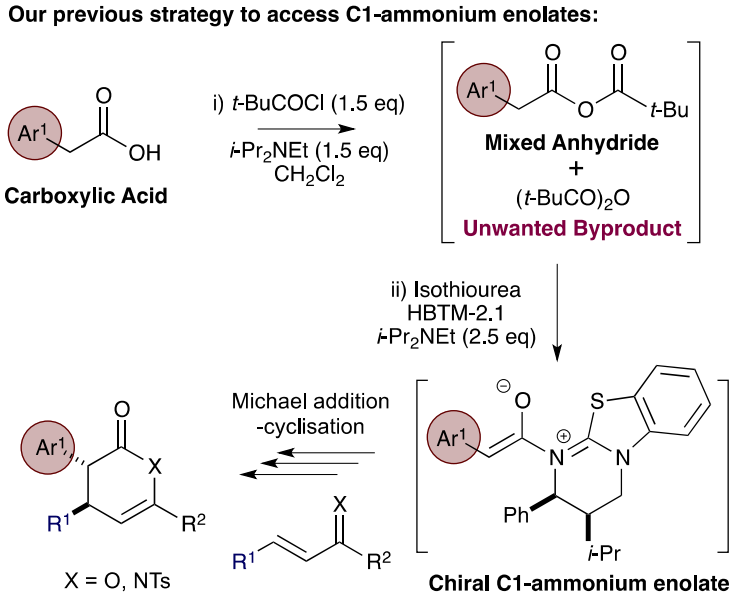

Figure 1. Previous access to isothiourea-derived C1-ammonium enolates from carboxylic acids

Using this strategy, the only by-product from such a process would be an equivalent of the parent acid that would be easily removed via basic aqueous work-up. While one equivalent of the parent arylacetic acid would be discarded in this process, their commercial availability and relatively cheap cost would mitigate their use in the asymmetric formation of diverse valueadded chemical building blocks. We showcase herein our results concerning the asymmetric Michael additionlactonisation of isothiourea derived ammonium enolates from readily prepared 2-arylacetic anhydrides and a range of Michael acceptors, giving stereodefined products with high diastereoand enantiocontrol (up to 95:5 dr and >99\% ee). ${ }^{16}$ 


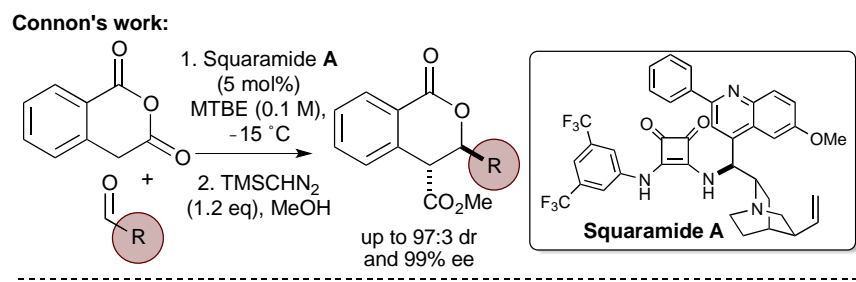

Chi's work:

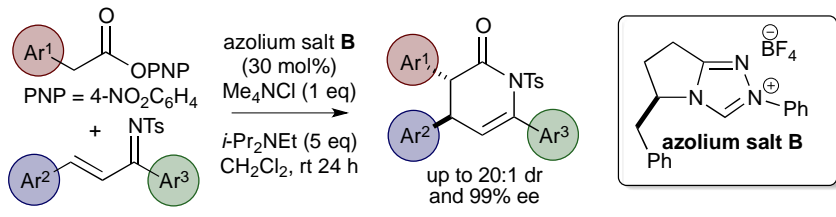

This work: preformed arylacetic anhydrides as precursors
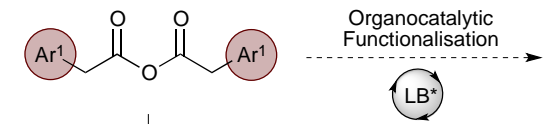

HBTM-2.1
Base (1 eq)
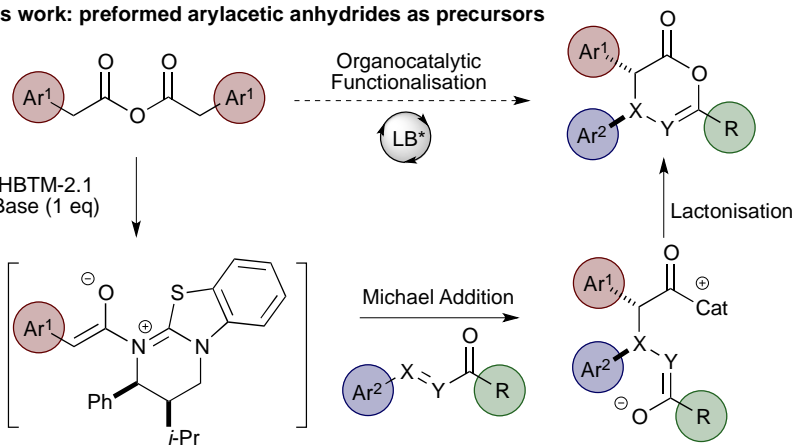

Figure 2. Proposed direct access to C1-ammonium enolates from arylacetic anhydrides.

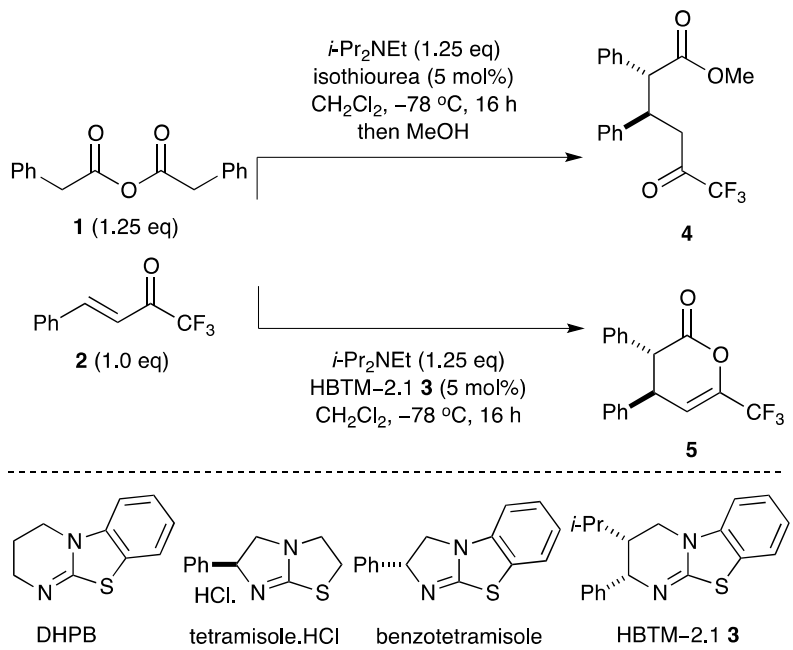

\begin{tabular}{|c|c|c|c|c|c|c|}
\hline Entry & Isothiourea & Prod & $\mathbf{T} /{ }^{\circ} \mathbf{C}$ & $\mathbf{d r}^{\mathbf{a}}$ & Yield & Ee $^{\mathbf{d}}$ \\
\hline 1 & DHPB & $\mathbf{4}$ & $\mathrm{rt}$ & $90: 10$ & $71^{\mathrm{b}}$ & \\
\hline 2 & tetramisole & $\mathbf{4}$ & $\mathrm{rt}$ & $87: 13$ & $52^{\mathrm{b}}$ & 83 \\
\hline 3 & benzotetramisole & $\mathbf{4}$ & $\mathrm{rt}$ & $84: 16$ & $58^{\mathrm{b}}$ & 71 \\
\hline 4 & HBTM-2.1 3 & $\mathbf{4}$ & $\mathrm{rt}$ & $80: 20$ & $67^{\mathrm{b}}$ & 88 \\
\hline 5 & HBTM-2.1 3 & $\mathbf{4}$ & -78 & $90: 10$ & $78^{\mathrm{b}}$ & 99 \\
\hline 6 & HBTM-2.1 3 & $\mathbf{5}$ & -78 & $94: 6$ & $81^{\mathrm{c}}$ & 98 \\
\hline
\end{tabular}

Table 1. Optimisation studies: [a] Determined by ${ }^{1} \mathrm{H}$ NMR spectroscopic analysis of the crude reaction mixture; [b] Isolated yield of $\mathbf{4}$ ( $\geq 95: 5 \mathrm{dr}$ ); [c] Isolated yield of 5 ( $90: 10 \mathrm{dr})$; [d] Determined by chiral HPLC analysis.

\section{Results and discussion}

\section{Optimisation Studies:}

Initial proof of concept studies used 2-phenylacetic anhydride $\mathbf{1}$ as an ammonium enolate precursor and trifluoromethylenone $\mathbf{2}$ as a Michael acceptor, with laboratory grade solvents employed as standard. 2-Phenylacetic anhydride (and all 2-arylacetic anhydrides used throughout this manuscript) is simply prepared by reaction of the parent arylacetic acid with DCC and is bench stable for approximately one week. ${ }^{17}$ Using achiral DHPB (10 $\mathrm{mol} \%$ ) as a catalyst only $\sim 80 \%$ conversion of trifluoromethyl ketone 2 was observed when 1 equivalent of both 2phenylacetic anhydride and $i$ - $\operatorname{Pr}_{2} \mathrm{NEt}$ were used. Using a slight excess of anhydride 1 (1.25 eq) and $i-\operatorname{Pr}_{2} \mathrm{NEt}(1.25 \mathrm{eq})$ was necessary for complete consumption of $\mathbf{2}$, presumably due to Claisen-type self condensation of the anhydride, giving 4 after in situ ring opening with $\mathrm{MeOH}$ in $71 \%$ yield and 90:10 dr at rt. Screening of a small number of chiral isothioureas in this process showed that HBTM-2.1 3 offered higher enantioselectivity then either tetramisole or benzotetramisole at rt (entries 2-4, Scheme 1). Lowering the reaction temperature to $-78{ }^{\circ} \mathrm{C}$ gave optimal diastereo- and enantiocontrol using HBTM-2.1 3 (entry 5), giving trifluoromethyl ketone 4 directly from anhydride $\mathbf{1}$ in $78 \%$ yield, $90: 10 \mathrm{dr}$ and $99 \%$ ee. Alternatively, Michael addition-lactonisation gave lactone $\mathbf{5}$ that was isolated in $81 \%$ yield and with excellent diastereo- and enantioselectivity (94:6 dr, 98\% ee). In all cases, highly pure material could be obtained after a simple acid/base work-up, with chromatographic purification used to obtain analytical samples.
Generality: Anhydrides as ammonium enolate precursors for asymmetric Michael addition-lactonisation with trifluoromethylenones

With an optimised process developed, the generality of this asymmetric Michael addition-lactonisation process using 2arylacetic anhydrides was investigated. Sequential variation of the anhydride component was first investigated using trifluoromethylenone 2 (Table 2), with all product racemates prepared using DHPB. Using $5 \mathrm{~mol} \%$ of 3 as standard, under optimised conditions this process readily tolerates 3- or 4-aryl substitution within the anhydride, including electronwithdrawing and -donating substituents (products 6-11) as well as extended aromatic systems (product 12). Interestingly, 2-aryl substitution within the anhyride is not tolerated in this system. For example the use of 2-(2-methylphenyl)acetyl 2-(2methylphenyl)acetate gave $<5 \%$ conversion to the desired product. Furthermore the use of 2-(thiophen-3-yl)acetic anhydride leads to reduced product diastereoselectivity (product 13), although still gives the major diastereoisomer in high ee (>99\% ee). 


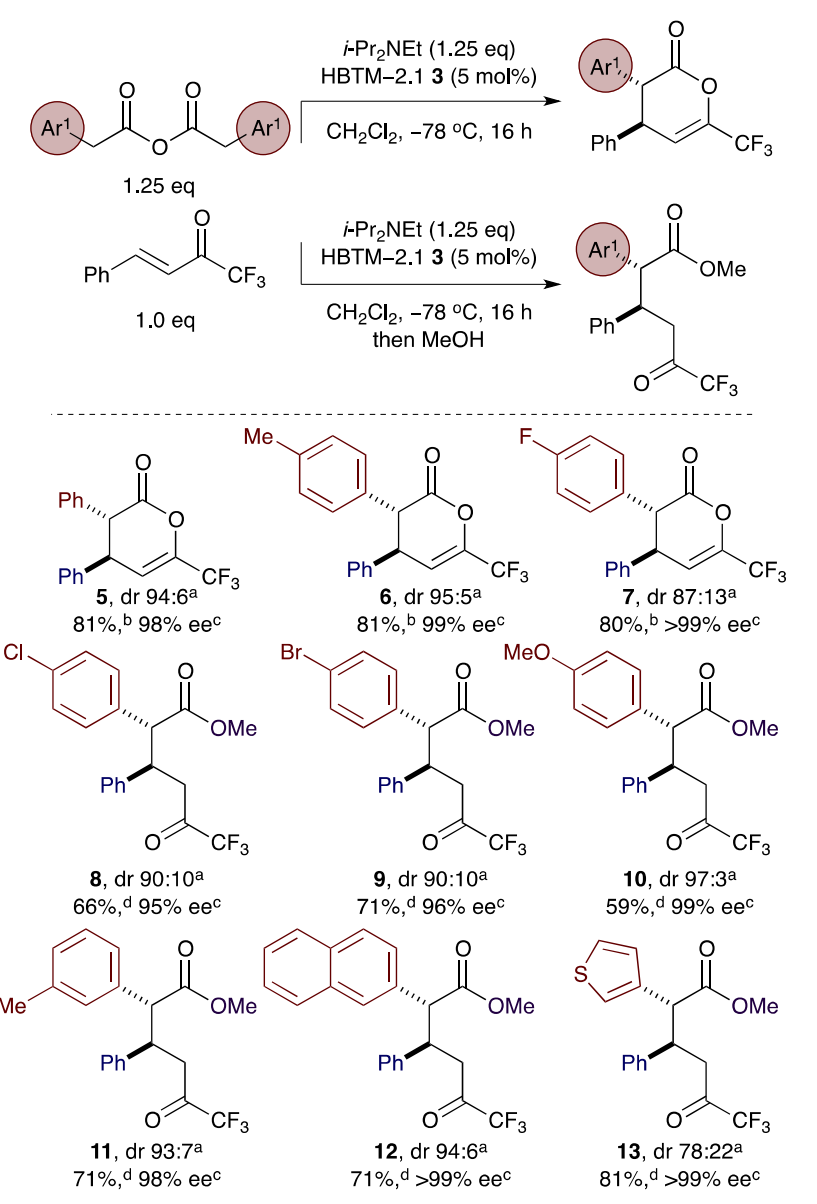

Table 2. Variation of arylacetic anhydride component; [a] Determined by ${ }^{1} \mathrm{H}$ NMR spectroscopic analysis of the crude reaction mixture; [b] Isolated yield ( $\geq 95: 5 \mathrm{dr}$ ); [c] Determined by chiral HPLC analysis; [d] Isolated yield at stated dr.

Subsequent studies showed the versatility of this process by focusing upon functionalisation of 2-phenylacetic anhydride with a range of trifluoromethylenones as well as variation of the nucleophilic ring-opening reaction component (Table 3). For example, in addition to in situ ring opening with methanol, alternative nucleophiles such as ethanol and allyl alcohol can also be used (products $\mathbf{1 4}$ and 15). Within the Michael acceptor, both electron-withdrawing (4-Cl) and electron-donating (4$\mathrm{OMe}$ ) substituents in the aryl unit are readily incorporated, providing the corresponding products in high ee. Heteroaryl substituents (2-furyl), as well as extended aromatic substituents (2-Np) can also be incorporated with good dr (up to 88:12) and ee (up to $99 \%$ ee).
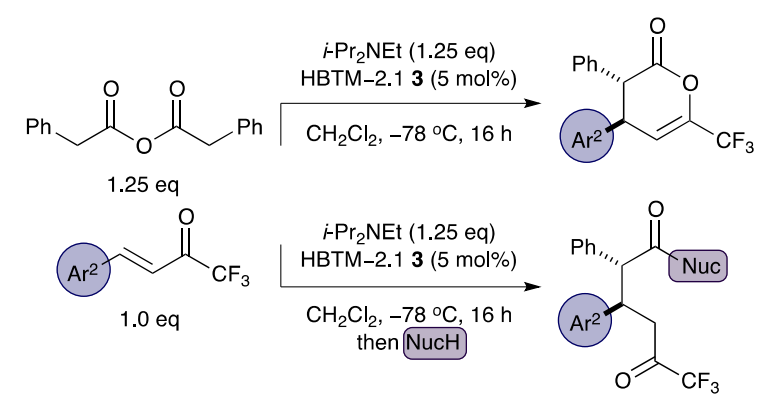<smiles>COC(=O)[C@H](c1ccccc1)C(CC(=O)C(F)(F)F)c1ccccc1</smiles><smiles>CCOC(=O)[C@H](c1ccccc1)C(CC(=O)C(F)(F)F)c1ccccc1</smiles>
14 , dr $85: 15^{\mathrm{a}}$ $84 \%,{ }^{b} 99 \%$ eec<smiles>COC(=O)[C@@H](c1ccccc1)C(CC(=O)C(F)(F)[18O][Ba])c1ccccc1</smiles>

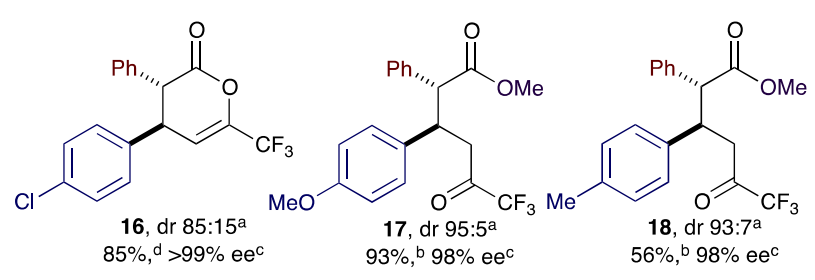<smiles>O=C1OC(C(F)(F)F)=C[C@H](c2ccc3ccccc3c2)[C@H]1c1ccccc1</smiles>

19 , dr $89: 11^{\mathrm{a}}$

$$
86 \% \text {, d } 99 \% \text { ee }^{c}
$$<smiles>COC(=O)[C@H](c1ccco1)[C@H](CC(=O)C(F)(F)F)c1ccccc1</smiles>

20, dr $88: 12^{a}$ $81 \%,{ }^{d}>99 \% e^{c}$
Table 3. Variation of trifluoromethylenone and nucleophilic component; [a] Determined by ${ }^{1} \mathrm{H}$ NMR spectroscopic analysis of the crude reaction mixture; [b] Isolated yield at stated dr. [c] Determined by chiral HPLC analysis; [d] Isolated yield ( $\geq 95: 5 \mathrm{dr})$.

\section{Anhydrides as ammonium enolate precursors for Michael addition-lactonisations with $\alpha$-keto- $\beta, \gamma$-unsaturated esters and $N$-aryl- $N$-aroyldiazenes}

Having developed an efficient protocol for the isothiourea catalysed Michael addition-lactonisation of anhydrides and 4aryl-trifluoromethylenones, this process was extended to other classes of suitably electron deficient Michael acceptors such as $\alpha$-keto- $\beta, \gamma$-unsaturated esters and $N$-aryl- $N$-aroyldiazenes. While DHPB can be used to prepare product racemates for analysis, $( \pm)$-HBTM-2.1 was used with these reaction classes (and is recommended from a practical standpoint) as it typically leads to a cleaner reaction profile and higher product yields. In the asymmetric series, $\alpha$-keto- $\beta, \gamma$-unsaturated esters proved suitable partners within this process (Table 4). Once more, electron-donating and electron-withdrawing substituents, as well as heteroaryl substitution within both anhydride and $\alpha$ keto- $\beta, \gamma$-unsaturated ester reaction components was tolerated, affording a range of anti-dihydropyranones in high yields (78$86 \%$ ) and with high diastereo- and enantioselectivity (up to 98:2 dr, exclusively $>99 \%$ ee). 


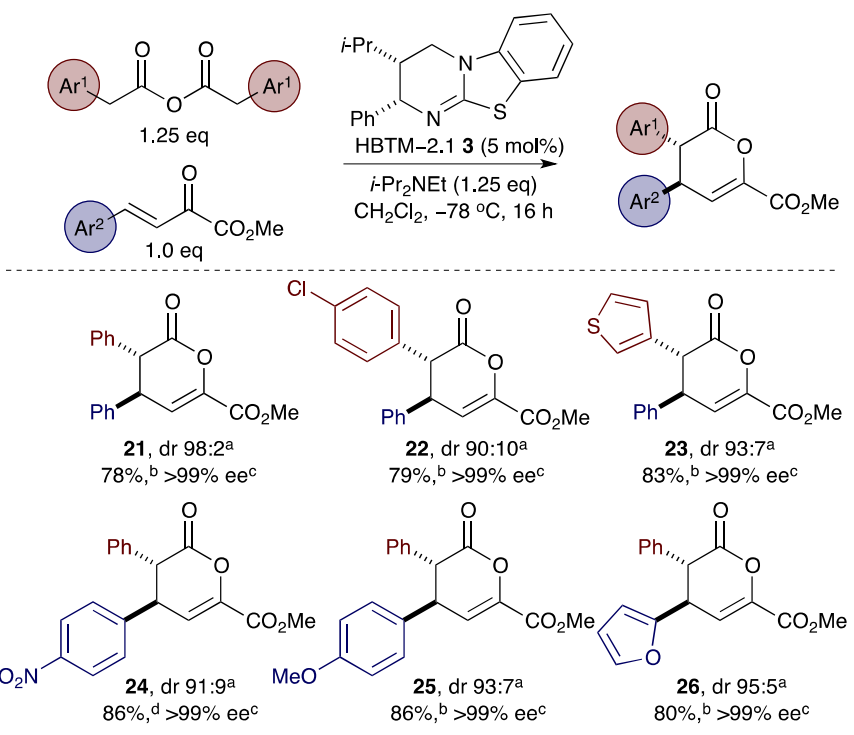

Table 4. Michael addition-lactonization using $\alpha$-keto- $\beta, \gamma$-unsaturated esters; [a] Determined by ${ }^{1} \mathrm{H}$ NMR spectroscopic analysis of the crude reaction mixture; [b] Isolated yield ( $\geq 98: 2 \mathrm{dr}$ ); [c] Determined by chiral HPLC analysis; [d] Isolated yield (93:7 dr).

As a final demonstration of the utility of this methodology, functionalisation of 2-arylacetic anhydrides with $\mathrm{N}$-aryl- $\mathrm{N}$ aroyldiazenes using a tandem Michael addition-lactonisation ring-opening protocol with $\mathrm{MeOH}$ was achieved (Table 5). Using simple reaction conditions, a range of anhydrides bearing electron-donating and electron-withdrawing aryl substituents, as well as heteroaryl substitution were tolerated, alongside variation of the acyl unit within the $N$-aryl- $N$-aroyldiazenes. In all cases, a variety of stereodefined hydrazides were isolated in high yields (61-85\%) and excellent enantioselectivity (up to $>99 \%$ ee) following in situ methanolysis.
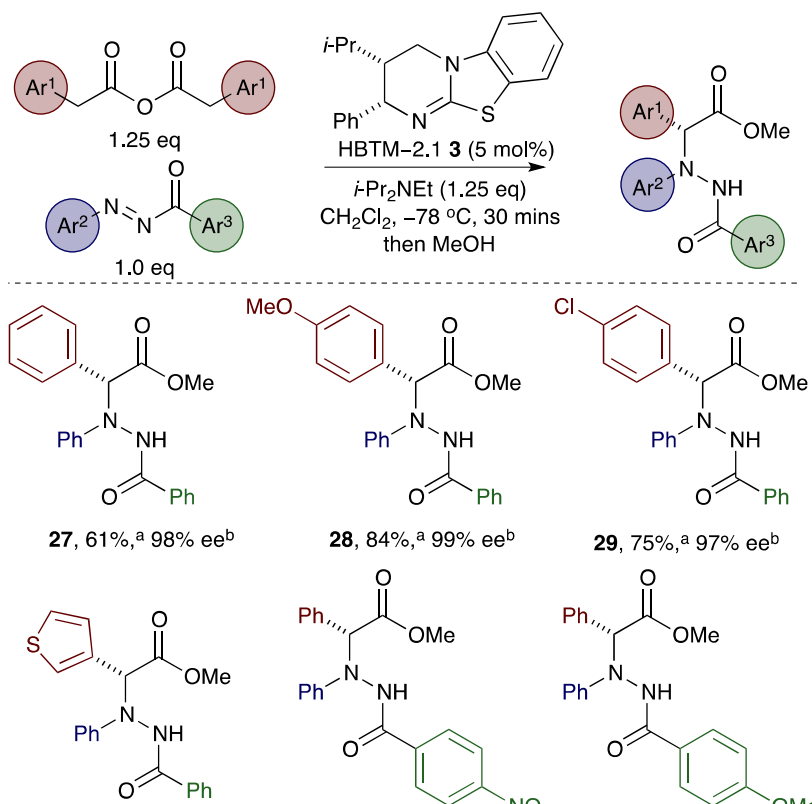

$28,84 \%,^{\text {a }} 99 \%$ ee

29, $75 \%$, a $97 \%$ ee

$30,75 \%$, $95 \%$ ee<smiles>COC(=O)[C@H](c1ccccc1)N(NC(=O)c1ccc([N+](=O)[O-])cc1)c1ccccc1</smiles><smiles>COC(=O)[C@H](c1ccccc1)N(NC(=O)c1ccc(OC)cc1)c1ccccc1</smiles>

31, $78 \%$, ${ }^{\text {a }} 99 \%$ ee $^{\text {b }}$

32, $78 \%$, ${ }^{\text {a }} 99 \%$ ee<smiles>COC(=O)[C@H](c1ccccc1)N(NC(=O)c1ccc(Cl)cc1)c1ccccc1</smiles>

33, $74 \%$, a $98 \% \mathrm{ee}^{\mathrm{b}}$<smiles>COC(=O)[C@H](c1ccccc1)N(NC(=O)c1ccc(C)cc1)c1ccccc1</smiles>

$34,85 \%,{ }^{\text {a }} 99 \%$ ee
Table 5. Michael addition-lactonization using $N$-aryl- $N$-aroyldiazenes; Isolated yield; [b] Determined by chiral HPLC analysis.

For all of these transformations we postulate a catalytic cycle that proceeds via initial $N$-acylation of HBTM-2.1 with the arylacetic anhydride to form the corresponding acyl ammonium ion. Deprotonation generates the corresponding $(Z)$-enolate, which undergoes stereoselective Michael addition, followed by intramolecular cyclisation, to generate the corresponding heterocyclic species (Figure 3). The sense of stereoinduction in these transformations is consistent with our previous rationale. $^{11 \mathrm{a}, \mathrm{c}}$ 


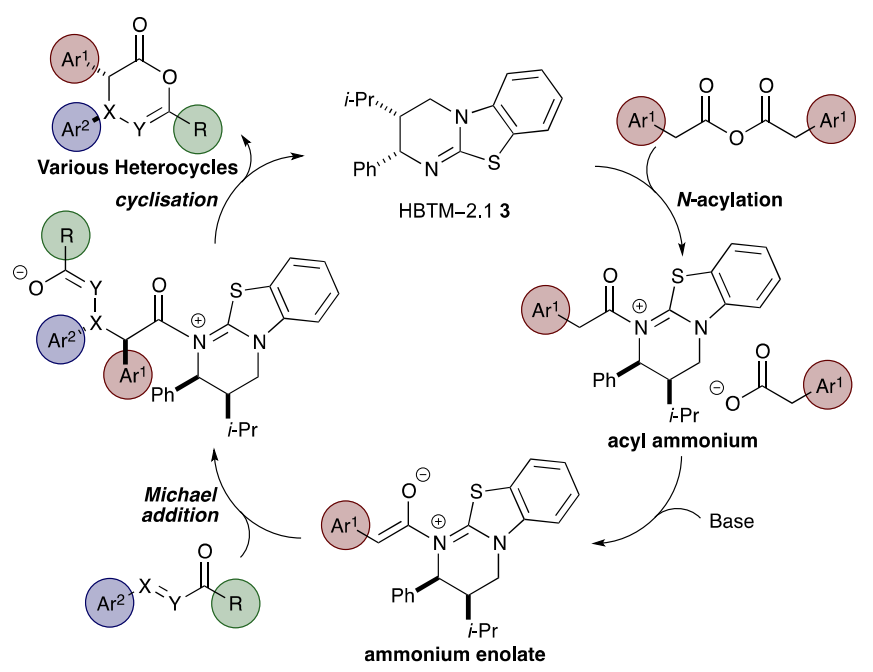

Figure 3. Proposed mechanism of asymmetric heterocycle formation.

\section{Conclusions}

In conclusion, 2-arylacetic anhydrides are convenient and readily prepared precursors for the formation of ammonium enolates in isothiourea-mediated Michael additionlactonisation processes. $N$-aryl- $N$-aroyldiazenes, 4-aryltrifluoromethylenones and $\alpha$-keto- $\beta, \gamma$-unsaturated esters are reactive Michael accceptors in this process, with HBTM-2.1 $(5 \mathrm{~mol} \%)$ readily promoting heterocycle formation with high diastereo- and enantiocontrol (up to 95:5 dr, up to $>99 \%$ ee). This protocol offers a useful and practical alternative to the in situ carboxylic acid activation method, in which by-product formation and the amount of sacrificial base used is minimised. Current research from this laboratory is directed toward developing alternative applications of isothioureas in asymmetric catalysis and expanding the synthetic utility of anhydrides as ammonium enolate precursors.

\section{Experimental}

\section{General Information}

Reactions involving moisture sensitive reagents were carried out under an argon atmosphere using standard vacuum line techniques in addition to freshly distilled solvents. All glassware used was flame dried and cooled under vacuum.

Solvents (THF, $\mathrm{CH}_{2} \mathrm{Cl}_{2}$, toluene, hexane and $\mathrm{Et}_{2} \mathrm{O}$ ) were obtained anhydrous and purified by an alumina column (Mbraun SPS-800). Petrol is defined as petroleum ether 40-60 ${ }^{\circ} \mathrm{C}$. All other solvents and commercial reagents were used as supplied without further purification unless stated otherwise. Room temperature (rt) refers to $20-25{ }^{\circ} \mathrm{C}$. Temperatures of 0 ${ }^{\circ} \mathrm{C}$ and $-78{ }^{\circ} \mathrm{C}$ were obtained using ice/water and $\mathrm{CO}_{2}(\mathrm{~s}) /$ acetone baths respectively. Temperatures of $0{ }^{\circ} \mathrm{C}$ to $50{ }^{\circ} \mathrm{C}$ for overnight reactions were obtained using an immersion cooler (HAAKE EK 90). Reflux conditions were obtained using an oil bath equipped with a contact thermometer. In vacuo refers to the use of a Büchi Rotavapor R-2000 rotary evaporator with a Vacubrand $\mathrm{CVC}_{2}$ vacuum controller or a Heidolph Laborota 4001 rotary evaporator with a vacuum controller.

Analytical thin layer chromatography was performed on precoated aluminium plates (Kieselgel $60 \mathrm{~F}_{254}$ silica). TLC visualisation was carried out with ultraviolet light $(254 \mathrm{~nm})$, followed by staining with a $1 \%$ aqueous $\mathrm{KMnO}_{4}$ solution. Flash column chromatography was performed on Kieselgel 60 silica in the solvent system stated.

${ }^{1} \mathrm{H}$ and ${ }^{13} \mathrm{C}$ nuclear magnetic resonance (NMR) spectra were acquired on either a Bruker Avance $300\left(300 \mathrm{MHz},{ }^{1} \mathrm{H}, 75\right.$ $\left.\mathrm{MHz}{ }^{13} \mathrm{C}\right)$, Bruker Avance II $400\left(400 \mathrm{MHz},{ }^{1} \mathrm{H}, 100 \mathrm{MHz}\right.$ ${ }^{13} \mathrm{C}$ ) or a Bruker Avance II $400\left(500 \mathrm{MHz},{ }^{1} \mathrm{H}, 125 \mathrm{MHz}{ }^{13} \mathrm{C}\right)$ spectrometer at ambient temperature in the deuterated solvent stated. All chemical shifts are quoted in parts per million (ppm) relative to the residual solvent as the internal standard. All coupling constants, $J$, are quoted in Hz. Multiplicities are indicated by: s (singlet), d (doublet), t (triplet), q (quartet), $\mathrm{ABq}$ ( $\mathrm{AB}$ quartet), sept (septet), oct (octet), m (multiplet), dd (doublet of doublets), ddd (doublet of doublet of doublets, $\mathrm{dt}$ (doublet of triplets) and td (triplet of doublets). The abbreviation Ar is used to denote aromatic, br to denote broad and app. to denote apparent.

Infrared spectra $\left(v_{\max }\right)$ were recorded on a Perkin-Elmer Spectrum GX FT-IR spectrometer using either thin films on $\mathrm{NaCl}$ plates or $\mathrm{KBr}$ discs. Only the characteristic peaks are quoted. Melting points were recorded on an Electrothermal apparatus and are uncorrected.

HPLC analyses were obtained on two separate machines; a Gilson HPLC consisting of a Gilson 305 pump, Gilson 306 pump, Gilson 811C dynamic mixer, Gilson 805 manometric module, Gilson 401C dilutor, Gilson 213XL sample injector and sample detection was performed with a Gilson 118 $\mathrm{UV} /$ vis detector while the temperature was assumed to be 20 ${ }^{\circ} \mathrm{C}$; a Shimadzu HPLC consisting of a DGU-20A5 degasser, LC-20AT liquid chromatograph, SIL-20AHT autosampler, CMB-20A communications bus module, SPD-M20A diode array detector and a CTO-20A column oven which allowed the temperature to be set from $25-40{ }^{\circ} \mathrm{C}$. Separation was achieved using Chiralcel OD-H and OJ-H columns or Chiralpak AD-H, AS-H, IA, IB, IC and ID columns.

Mass spectrometry $(\mathrm{m} / \mathrm{z})$ data were acquired by electrospray ionisation (ES), electron impact (EI) or nanospray ionisation (NSI) either at the University of St Andrews or the EPSRC National Mass Spectrometry Service Centre, Swansea. At the University of St Andrews, low and high resolution ESI MS were carried out on a Micromass LCT spectrometer. At the EPSRC National Mass Spectrometry Service Centre, low resolution NSI MS was carried out on a Micromass Quattro II spectrometer and high resolution NSI MS on a Thermofisher LTQ Orbitrap XL spectrometer.

Optical rotations were measured on a Perkin Elmer Precisely/Model-341 polarimeter operating at the sodium D line with a $100 \mathrm{~mm}$ path cell. 
General procedure A: Formation of anhydrides.

To a solution of carboxylic acid (1 equiv.) in toluene was added DCC $(0.50-0.55 \mathrm{eq})$ and the solution was allowed to stir at $\mathrm{rt}$ for 15 minutes. The reaction mixture was filtered and the filtrate was concentrated in vacuo to give the crude reaction mixture.

General procedure B: Michael-lactonisations.

To a solution of anhydride (1.25 equiv.) in $\mathrm{CH}_{2} \mathrm{Cl}_{2}(\sim 2 \mathrm{~mL}$ per $0.2 \mathrm{mmol}$ of anhydride) was added either $( \pm)$-HBTM-2.1 or (2S,3R)-HBTM-2.1 (5 mol\%) followed by Michael acceptor ( 1 equiv.) and DIPEA (1.25 equiv.) at $-78{ }^{\circ} \mathrm{C}$. The reaction mixture was stirred at $-78^{\circ} \mathrm{C}$ until complete by TLC and was subsequently quenched by addition of $1 \mathrm{M} \mathrm{HCl}$. Once warmed to rt, the reaction mixture was poured into water and extracted twice with $\mathrm{CH}_{2} \mathrm{Cl}_{2}$. The combined organics were washed with sat. aq. $\mathrm{NaHCO}_{3}$, dried $\left(\mathrm{MgSO}_{4}\right)$, filtered and concentrated in vacuo to give the crude reaction mixture.

General Procedure C: Tandem Michael-lactonisation ringopening (HBTM-2.1)

To a solution of anhydride (1.25 equiv.) in $\mathrm{CH}_{2} \mathrm{Cl}_{2}(\sim 2 \mathrm{~mL}$ per $0.2 \mathrm{mmol}$ of anhydride) was added either ( \pm )-HBTM-2.1 or (2S,3R)-HBTM-2.1 (5 mol\%) followed by Michael acceptor ( 1 equiv.) and DIPEA (1.25 equiv.) at $-78{ }^{\circ} \mathrm{C}$. The reaction mixture was stirred at the required temperature until complete by TLC then excess alcohol was added. This was stirred overnight at $\mathrm{rt}$. The reaction mixture was quenched by addition of $1 \mathrm{M} \mathrm{HCl}$. Once warmed to $\mathrm{rt}$, the reaction mixture was poured into water and extracted twice with $\mathrm{CH}_{2} \mathrm{Cl}_{2}$. The combined organics were washed with sat. aq. $\mathrm{NaHCO}_{3}$, dried $\left(\mathrm{MgSO}_{4}\right)$, filtered and concentrated in vacuo to give the crude reaction mixture.

\section{Starting Materials Used Isothiourea Catalysts}

3,4-dihydro-2 $H$-pyrimido[2,1-b]benzothiazole (DHPB) 35, HBTM-2.1 $( \pm) \mathbf{3}$ and HBTM-2.1 $(2 S, 3 R) \mathbf{3}$ were made according to literature procedures. ${ }^{11 \mathrm{e}}$

\section{Trifluoromethyl Enones}

(E)-1,1,1-trifluoro-4-phenylbut-3-en-2-one chlorophenyl)-1,1,1-trifluorobut-3-en-2-one trifluoro-4-(naphthalen-2-yl)but-3-en-2-one methylphenyl)-1,1,1-trifluorobut-3-en-2-one bromophenyl)-1,1,1-trifluorobut-3-en-2-one methoxyphenyl)-1,1,1-trifluorobut-3-en-2-one 1,1,1-trifluoro-4-(furan-2-yl)but-3-en-2-one $\mathbf{4 1}$ were according to literature procedures. ${ }^{11 \mathrm{e}}$

\section{$\alpha$-keto- $\beta, \gamma$-unsaturated esters used}

methyl (3E)-2-oxo-4-phenylbut-3-enoate 42, methyl (3E)-4(4-nitrophenyl)-2-oxobut-3-enoate 43, methyl (3E)-4-(4methoxyphenyl)-2-oxobut-3-enoate $\mathbf{4 4}$ and methyl (3E)-4(furan-2-yl)-2-oxobut-3-enoate $\mathbf{4 5}$ were made according to literature procedures. ${ }^{11 \mathrm{a}}$

\section{$\mathrm{N}$-aryl- $\mathrm{N}$-aroyldiazenes used}

(E)-N-(phenylimino)benzamide (phenylimino)benzamide (phenylimino)benzamide (phenylimino)benzamide (phenylimino)benzamide literature procedures. ${ }^{11 \mathrm{c}}$

$\begin{array}{lr}\text { 46, } & (E)-4 \text {-chloro-N- } \\ \mathbf{4 7}, & (E)-4 \text {-nitro-N- } \\ \mathbf{4 8}, & (E)-4 \text {-methyl-N- }\end{array}$
49 and (E)-4-methoxy-N50 were made according to

\section{Preparation of anhydrides}

2-phenylacetic anhydride 1. Following general procedure A, phenylacetic acid $(1.00 \mathrm{~g}, 7.34 \mathrm{mmol})$, DCC $(0.83 \mathrm{~g}, 4.04$ mmol) and toluene $(20 \mathrm{~mL})$ gave anhydride 1 as a white solid (1.68 g, 90\%); mp 68-70 ${ }^{\circ} \mathrm{C} ;\left\{\right.$ lit. $\left.{ }^{18} \mathrm{mp} 72-72.5^{\circ} \mathrm{C}\right\} ; \delta_{\mathrm{H}}(400$ $\left.\mathrm{MHz}, \mathrm{CDCl}_{3}\right) 3.76\left(4 \mathrm{H}, \mathrm{s}, 2 \mathrm{CH}_{2}\right), 7.23-7.25(4 \mathrm{H}, \mathrm{m}, \mathrm{Ar} H)$, 7.32-7.38 (6H, m, $\mathrm{ArH})$. Spectroscopic data are in accordance with the literature. ${ }^{19}$

2-(p-tolyl)acetic anhydride 51. Following general procedure A, $p$-tolylacetic acid $(1.00 \mathrm{~g}, 6.66 \mathrm{mmol}), \mathrm{DCC}(0.70 \mathrm{~g}, 3.40$ mmol) and toluene $(20 \mathrm{~mL})$ gave anhydride $\mathbf{5 1}$ as a white solid $(1.00 \mathrm{~g}, 53 \%)$; mp $47-49{ }^{\circ} \mathrm{C}$; $\left\{\right.$ lit. $\left.{ }^{19} \mathrm{mp} 56-57{ }^{\circ} \mathrm{C}\right\} ; \delta_{\mathrm{H}}$ $\left(300 \mathrm{MHz}, \mathrm{CDCl}_{3}\right) 2.39\left(6 \mathrm{H}, \mathrm{s}, \mathrm{CH}_{3}\right), 3.72\left(4 \mathrm{H}, \mathrm{s}, \mathrm{CH}_{2}\right)$, 7.12-7.19 $(8 \mathrm{H}, \mathrm{m}, \mathrm{Ar} H)$. Spectroscopic data are in accordance with the literature. ${ }^{20}$

2-(4-fluorophenyl)acetic anhydride 52. Following general procedure A, 4-fluorophenylacetic acid (1.00 g, $6.49 \mathrm{mmol})$, DCC $(0.74 \mathrm{~g}, 3.57 \mathrm{mmol})$ and toluene $(20 \mathrm{~mL})$ gave anhydride $\mathbf{5 2}$ as a white solid $(1.48 \mathrm{~g}, 79 \%)$; mp $36-38{ }^{\circ} \mathrm{C}$; $v_{\max }(\mathrm{KBr}) 3073,2919(\mathrm{C}-\mathrm{H}), 1821,1750(\mathrm{C}=\mathrm{O}), 1612,1511$; $\delta_{\mathrm{H}}\left(400 \mathrm{MHz}, \mathrm{CDCl}_{3}\right) 3.70\left(4 \mathrm{H}, \mathrm{s}, 2 \mathrm{CH}_{2}\right), 6.99-7.04(4 \mathrm{H}, \mathrm{m}$, $2 \operatorname{Ar}(3,5) H), 7.16-7.19(4 \mathrm{H}, \mathrm{m}, 2 \operatorname{Ar}(2,6) H) ; \delta_{\mathrm{C}}(100 \mathrm{MHz}$, $\left.\mathrm{CDCl}_{3}\right) 41.3\left(2 \mathrm{CH}_{2}\right), 115.8(\mathrm{~d}, J 21.4,2 \operatorname{ArC}(3,5)), 127.7$ (d, $J$ 3.1, $2 \operatorname{ArC}(1)$ ), 131.1 (d, $J 8.1,2 \operatorname{ArC}(2,6)), 162.4(\mathrm{~d}, J 245$, $2 \operatorname{ArC}(4)), 166.8(2 C=\mathrm{O}) ; \delta_{\mathrm{F}}\left(376 \mathrm{MHz}, \mathrm{CDCl}_{3}\right)-115.1(\mathrm{Ar} F)$; $\begin{array}{lllll}m / z & \left(\mathrm{ES}^{+}\right) & 313 & \left([\mathrm{M}+\mathrm{Na}]^{+},\right. & 100 \%)\end{array}$ $\mathrm{C}_{16} \mathrm{H}_{12} \mathrm{~F}_{2} \mathrm{NaO}_{3}^{+} \quad\left([\mathrm{M}+\mathrm{Na}]^{+}\right)$requires 313.0652; found 313.0655 (+1.0 ppm).

2-( $\boldsymbol{m}$-tolyl)acetic anhydride 53. Following general procedure A, $m$-tolylacetic acid $(1.00 \mathrm{~g}, 6.62 \mathrm{mmol}), \mathrm{DCC}(0.72 \mathrm{~g}, 3.50$ mmol) and toluene $(20 \mathrm{~mL})$ gave anhydride $\mathbf{5 3}$ as a yellow oil $(0.75 \mathrm{~g}, 80 \%) ; \delta_{\mathrm{H}}\left(300 \mathrm{MHz}, \mathrm{CDCl}_{3}\right) 2.27\left(6 \mathrm{H}, \mathrm{s}, \mathrm{CH}_{3}\right), 3.54$ $\left(4 \mathrm{H}, \mathrm{s}, 2 \mathrm{CH}_{2}\right), 6.99-7.02(3 \mathrm{H}, \mathrm{m}, \mathrm{Ar} H), 7.12-7.18(5 \mathrm{H}, \mathrm{m}$, $\mathrm{ArH})$. Spectroscopic data are in accordance with the literature. $^{20}$

2-(4-bromophenyl)acetic anhydride 54. Following general procedure A, 4-bromophenylacetic acid (1.00 g, $4.65 \mathrm{mmol})$, DCC $(0.51 \mathrm{~g}, 2.46 \mathrm{mmol})$ and toluene $(20 \mathrm{~mL})$ gave anhydride 54 as a white solid $(0.95 \mathrm{~g}, 98 \%)$; mp 76-78 ${ }^{\circ} \mathrm{C} ;{ }^{21}$ $\delta_{\mathrm{H}}\left(300 \mathrm{MHz}, \mathrm{CDCl}_{3}\right) 3.61\left(4 \mathrm{H}, \mathrm{s}, 2 \mathrm{CH}_{2}\right), 6.98-7.02(4 \mathrm{H}, \mathrm{m}$, $2 \operatorname{Ar}(3,5) H), 7.36-7.41(4 \mathrm{H}, \mathrm{m}, 2 \operatorname{Ar}(2,6) H)$. Spectroscopic data are in accordance with the literature. ${ }^{22}$ 
2-(4-chlorophenyl)acetic anhydride 55. Following general procedure A, 4-chlorophenylacetic acid (1.00 g, $5.90 \mathrm{mmol})$, DCC $(0.60 \mathrm{~g}, 2.90 \mathrm{mmol})$ and toluene $(50 \mathrm{~mL})$ gave anhydride 55 as a light yellow solid (0.96 g, quant.); mp 62$64{ }^{\circ} \mathrm{C} ; v_{\max }(\mathrm{KBr}) 3482,3038,2908(\mathrm{C}-\mathrm{H}), 1801(\mathrm{C}=\mathrm{O}), 1753$ $(\mathrm{C}=\mathrm{O}), 1598,1491,1402,1338,1213,752(\mathrm{C}-\mathrm{Cl}) ; \delta_{\mathrm{H}}(500$ $\left.\mathrm{MHz}, \mathrm{CDCl}_{3}\right) 3.70\left(4 \mathrm{H}, \mathrm{s}, 2 \mathrm{CH}_{2}\right), 7.13(4 \mathrm{H}, \mathrm{d}, J 8.5$, $2 \operatorname{Ar}(3,5) H), 7.28-7.31(4 \mathrm{H}, \mathrm{m}, 2 \operatorname{Ar}(2,6) H) ; \delta_{\mathrm{C}}(100 \mathrm{MHz}$, $\left.\mathrm{CDCl}_{3}\right) 41.5\left(2 \mathrm{CH}_{2}\right), 129.1(2 \mathrm{ArC}), 130.4(2 \operatorname{ArC}(1)), 130.8$ (2ArC), $133.9(2 \operatorname{ArC}(4)), 166.5 \quad(2 C=\mathrm{O}) ; \mathrm{m} / z \quad\left(\mathrm{ES}^{+}\right) 345$ $\left([\mathrm{M}+\mathrm{Na}]^{+}, 100 \%\right)$; HRMS $\left(\mathrm{ES}^{+}\right) \mathrm{C}_{16} \mathrm{H}_{12} \mathrm{Cl}_{2} \mathrm{NaO}_{3}\left([\mathrm{M}+\mathrm{Na}]^{+}\right)$ requires 345.0061 ; found 345.0055 (-1.8 ppm).

2-(4-methoxyphenyl)acetic anhydride 56. Following general procedure A, 4-methoxyphenylacetic acid $(1.00 \mathrm{~g}$, $6.00 \mathrm{mmol})$, DCC (0.62 g, $3.00 \mathrm{mmol})$ and toluene $(25 \mathrm{~mL})$ gave anhydride 56 as a white solid (0.95 g, quant.); mp 60-62 ${ }^{\circ} \mathrm{C} ; v_{\max }(\mathrm{KBr}) 2837(\mathrm{C}-\mathrm{H}), 1798(\mathrm{C}=\mathrm{O}), 1736(\mathrm{C}=\mathrm{O}), 1611$, $1510,1300,1242(\mathrm{C}-\mathrm{O}) ; \delta_{\mathrm{H}}\left(300 \mathrm{MHz}, \mathrm{CDCl}_{3}\right) 3.66(4 \mathrm{H}, \mathrm{s}$, $\left.2 \mathrm{CH}_{2}\right), 3.80\left(6 \mathrm{H}, \mathrm{s}, 2 \mathrm{CH}_{3}\right), 6.83-6.86(4 \mathrm{H}, \mathrm{m}, 2 \mathrm{Ar}(3,5) H)$, 7.10-7.13 (4H, m, $2 \operatorname{Ar}(2,6) H) ; \delta_{\mathrm{C}}\left(75 \mathrm{MHz}, \mathrm{CDCl}_{3}\right) 41.4$ $\left(2 \mathrm{CH}_{2}\right), 55.4\left(2 \mathrm{CH}_{3}\right), 114.3(2 \mathrm{ArC}(3,5)), 124.1(2 \mathrm{ArC}(1))$, $130.6(2 A r C(2,6)), 159.2(2 A r C(4)), 167.5(2 C=0) ; m / z\left(\mathrm{ES}^{+}\right)$ $337\left([\mathrm{M}+\mathrm{Na}]^{+}, 100 \%\right)$; HRMS $\left(\mathrm{ES}^{+}\right) \mathrm{C}_{18} \mathrm{H}_{18} \mathrm{NaO}_{5}\left([\mathrm{M}+\mathrm{Na}]^{+}\right)$ requires 337.1061; found $337.1052(+2.8 \mathrm{ppm})$.

2-(naphthalen-2-yl)acetic anhydride 57. Following general procedure A, 2-naphthylacetic acid $(1.00 \mathrm{~g}, 5.40 \mathrm{mmol})$, DCC $(0.56 \mathrm{~g}, 2.70 \mathrm{mmol})$ and toluene $(25 \mathrm{~mL})$ gave anhydride 57 as a white solid $(0.59 \mathrm{~g}, 61 \%)$; $\mathrm{mp} 104-108{ }^{\circ} \mathrm{C}$; $v_{\max }(\mathrm{KBr})$ 3055, $2928(\mathrm{C}-\mathrm{H}), 2116,1809(\mathrm{C}=\mathrm{O}), 1748(\mathrm{C}=\mathrm{O}), 1601$, 1508,$1325 ; \delta_{\mathrm{H}}\left(300 \mathrm{MHz}, \mathrm{CDCl}_{3}\right) 3.88\left(4 \mathrm{H}, \mathrm{s}, 2 \mathrm{CH}_{2}\right), 7.27$ $(2 \mathrm{H}, \mathrm{d}, J 7.4, \operatorname{Ar} H), 7.47-7.51(4 \mathrm{H}, \mathrm{m}, \operatorname{Ar} H), 7.63(2 \mathrm{H}, \mathrm{s}$, $2 \operatorname{Ar}(1) H), 7.70-7.73(4 \mathrm{H}, \mathrm{m}, \operatorname{Ar} H), 7.80-7.83(2 \mathrm{H}, \mathrm{m}, \operatorname{Ar} H)$; $\delta_{\mathrm{C}}\left(75 \mathrm{MHz}, \mathrm{CDCl}_{3}\right) 42.4\left(2 \mathrm{CH}_{2}\right), 126.2(2 \mathrm{ArC}), 126.5$ (2ArC), $127.1(2 A r C), 127.8(2 A r C), 127.8(2 A r C), 128.5$ (2ArC), $128.6(2 A r C), 129.5$ (4ry $2 A r C$ ), 132.7 (4ry $2 A r C$ ), $133.4(4 \mathrm{ry} 2 \mathrm{ArC}), 167.0(2 \mathrm{C}=\mathrm{O}) ; \mathrm{m} / \mathrm{z}\left(\mathrm{CI}^{+}\right) 372\left(\left[\mathrm{M}+\mathrm{NH}_{4}\right]^{+}\right.$, $100 \%)$; HRMS $\left(\mathrm{CI}^{+}\right) \quad \mathrm{C}_{24} \mathrm{H}_{22} \mathrm{NO}_{3}^{+}\left(\left[\mathrm{M}+\mathrm{NH}_{4}\right]^{+}\right)$requires 372.1594; found 372.1599 (+1.3 ppm).

2-(thiophen-3-yl)acetic anhydride 58. Following general procedure A, 3-thiophene acetic acid $(1.00 \mathrm{~g}, 7.00 \mathrm{mmol})$, DCC $(0.73 \mathrm{~g}, 3.50 \mathrm{mmol})$ and toluene $(25 \mathrm{~mL})$ gave anhydride 58 as a yellow solid $\left(0.96 \mathrm{~g}\right.$, quant.); mp $40-42{ }^{\circ} \mathrm{C}$; $v_{\max }(\mathrm{KBr}) 3098,2930(\mathrm{C}-\mathrm{H}), 1811(\mathrm{C}=\mathrm{O}), 1740(\mathrm{C}=\mathrm{O})$, $1539,1412,1330 ; \delta_{\mathrm{H}}\left(400 \mathrm{MHz}, \mathrm{CDCl}_{3}\right) 3.79\left(4 \mathrm{H}, \mathrm{s}, 2 \mathrm{CH}_{2}\right)$, $6.99(2 \mathrm{H}, \mathrm{dd}, J 5.0,1.2,2 \operatorname{Ar}(4) H), 7.15(2 \mathrm{H}, \mathrm{dd}, J 2.0,1.0$, $2 \operatorname{Ar}(2) H), 7.31(2 \mathrm{H}$, dd, $J 5.0,3.0,2 \operatorname{Ar}(5) H) ; \delta_{\mathrm{C}}(75 \mathrm{MHz}$, $\left.\mathrm{CDCl}_{3}\right) 36.7\left(2 \mathrm{CH}_{2}\right), 123.9(2 \mathrm{ArC}), 126.4(2 \mathrm{ArC}), 128.4$ $(2 \operatorname{ArC}), 131.5(2 \operatorname{ArC}(3)), 166.6(2 C=\mathrm{O}) ; \mathrm{m} / z \quad\left(\mathrm{ES}^{+}\right) 289$ $\left([\mathrm{M}+\mathrm{Na}]^{+}, 50 \%\right) ; \operatorname{HRMS}\left(\mathrm{ES}^{+}\right) \mathrm{C}_{12} \mathrm{H}_{10} \mathrm{NaO}_{3} \mathrm{~S}_{2}\left([\mathrm{M}+\mathrm{Na}]^{+}\right)$ requires 288.9969 ; found $288.9976(+2.5 \mathrm{ppm})$.

\section{Preparation of products (Table 2)}

(3R,4R)-3,4-diphenyl-6-(trifluoromethyl)-3,4-dihydro-2Hpyran-2-one 5. Following general procedure $\mathrm{B}$, anhydride 1 (63.5 mg, $0.25 \mathrm{mmol})$ in $\mathrm{CH}_{2} \mathrm{Cl}_{2}(2 \mathrm{~mL})$, HBTM-2.1 $(2 S, 3 R)-$ 3 (3.09 mg, $0.01 \mathrm{mmol}, 5 \mathrm{~mol} \%)$, enone 2 ( $40.0 \mathrm{mg}, 0.20$ mmol) and DIPEA $(43.5 \mu \mathrm{L}, 0.25 \mathrm{mmol})$ for $16 \mathrm{~h}$ at $-78{ }^{\circ} \mathrm{C}$ gave crude lactone $(3 R, 4 R)-5(94: 6 \mathrm{dr})$. Chromatographic purification (eluent $\mathrm{Et}_{2} \mathrm{O}$ :petrol 3.5:96.5) gave lactone $(3 R, 4 R)-5(>99: 1 \mathrm{dr})$ as a white solid $(51.8 \mathrm{mg}, 81 \%)$ with identical spectroscopic data as previously reported; ${ }^{11 \mathrm{e}}[\alpha]_{\mathrm{D}}{ }^{20}$ 219.2 (c 0.2, $\left.\mathrm{CH}_{2} \mathrm{Cl}_{2}\right)$; $\left\{\right.$ lit. $\left.{ }^{11 \mathrm{e}}[\alpha]_{\mathrm{D}}{ }^{20}-227.2(c) 0.25, \mathrm{CH}_{2} \mathrm{Cl}_{2}\right)$ for $99 \% e e$; Chiral HPLC Chiralpak AD-H (5\% IPA:hexane, flow rate $\left.1 \mathrm{~mL} \mathrm{~min}^{-1}, 211 \mathrm{~nm}, 20{ }^{\circ} \mathrm{C}\right) \mathrm{t}_{\mathrm{R}}(3 R, 4 R): 10.9 \mathrm{~min}$, $\mathrm{t}_{\mathrm{R}}(3 S, 4 S): 12.2 \mathrm{~min}, 98 \%$ ee.

(3R,4R)-4-phenyl-3-(p-tolyl)-6-(trifluoromethyl)-3,4dihydro-2H-pyran-2-one 6. Following general procedure $\mathrm{B}$, anhydride 51 (70.5 mg, $0.25 \mathrm{mmol})$ in $\mathrm{CH}_{2} \mathrm{Cl}_{2}(2 \mathrm{~mL})$, HBTM-2.1 (2S,3R)-3 (3.09 mg, $0.01 \mathrm{mmol}, 5 \mathrm{~mol} \%)$, enone 2 (40.0 mg, $0.20 \mathrm{mmol})$ and DIPEA $(43.5 \mu \mathrm{L}, 0.25 \mathrm{mmol})$ for $16 \mathrm{~h}$ at $-78{ }^{\circ} \mathrm{C}$ gave crude lactone $(3 R, 4 R)-6(95: 5 \mathrm{dr})$. Chromatographic purification (eluent $\mathrm{Et}_{2} \mathrm{O}$ :petrol 3:97) gave lactone $(3 R, 4 R)-6(>99: 1 \mathrm{dr})$ as a white solid $(51.8 \mathrm{mg}, 81 \%)$ with identical spectroscopic data as previously reported; ${ }^{11 \mathrm{e}}$ Chiral HPLC Chiralpak AD-H (2\% IPA:hexane, flow rate 1 $\left.\mathrm{mL} \min ^{-1}, 211 \mathrm{~nm}, 20{ }^{\circ} \mathrm{C}\right) \mathrm{t}_{\mathrm{R}}(3 S, 4 S): 14.0 \mathrm{~min}, \mathrm{t}_{\mathrm{R}}(3 R, 4 R)$ : $16.4 \mathrm{~min}, 99 \%$ ee.

(3R,4R)-3-(4-fluorophenyl)-4-phenyl-6-(trifluoromethyl)3,4-dihydro-2H-pyran-2-one 7. Following general procedure $\mathrm{B}$, anhydride $\mathbf{5 2}(72.5 \mathrm{mg}, 0.25 \mathrm{mmol})$ in $\mathrm{CH}_{2} \mathrm{Cl}_{2}(2 \mathrm{~mL})$, HBTM-2.1 (2S,3R)-3 (3.09 mg, $0.01 \mathrm{mmol}, 5 \mathrm{~mol} \%)$, enone 2 (40.0 mg, $0.20 \mathrm{mmol})$ and DIPEA $(43.5 \mu \mathrm{L}, 0.25 \mathrm{mmol})$ for $16 \mathrm{~h}$ at $-78{ }^{\circ} \mathrm{C}$ gave crude lactone $(3 R, 4 R)-7(87: 13 \mathrm{dr})$. Chromatographic purification (eluent $\mathrm{Et}_{2} \mathrm{O}$ :petrol 5:95) gave lactone $(3 R, 4 R)-7(>99: 1 \mathrm{dr})$ as a white solid $(54.0 \mathrm{mg}, 80 \%)$ with identical spectroscopic data as previously reported $;{ }^{11 \mathrm{e}}$ Chiral HPLC Chiralcel OD-H (10\% IPA:hexane, flow rate 1 $\left.\mathrm{mL} \min ^{-1}, 211 \mathrm{~nm}, 20{ }^{\circ} \mathrm{C}\right) \mathrm{t}_{\mathrm{R}}(3 R, 4 R): 19.2 \mathrm{~min}, \mathrm{t}_{\mathrm{R}}(3 S, 4 S)$ : $39.4 \mathrm{~min},>99 \%$ ee.

(2R,3R)-methyl 2-(4-chlorophenyl)-6,6,6-trifluoro-5-oxo-3phenylhexanoate 8 . Following general procedure $C$, anhydride 55 ( $80.8 \mathrm{mg}, 0.25 \mathrm{mmol})$, enone $2(40.0 \mathrm{mg}, 0.20$ mmol), HBTM-2.1 (2S,3R)-3 (3.08 mg, $0.01 \mathrm{mmol}, 5 \mathrm{~mol} \%)$, DIPEA $(43.5 \mu \mathrm{L}, 0.25 \mathrm{mmol})$ in $\mathrm{CH}_{2} \mathrm{Cl}_{2}(2 \mathrm{~mL})$ for $16 \mathrm{~h}$ at $78{ }^{\circ} \mathrm{C}$ followed by methanol $(2 \mathrm{~mL})$ gave $(2 R, 3 R)-8(90: 10$ dr). Chromatographic purification $\left(5 \% \mathrm{Et}_{2} \mathrm{O}\right.$ :petrol eluent) gave pure product $(2 R, 3 R)-\mathbf{8}(90: 10 \mathrm{dr})$ as a white solid (50.7 $\mathrm{mg}, 66 \%)$; mp $68-70{ }^{\circ} \mathrm{C} ;[\alpha]_{\mathrm{D}}{ }^{20}-102.2\left(c 0.5, \mathrm{CH}_{2} \mathrm{Cl}_{2}\right)$; Chiral HPLC Chiralpak IA (10\% IPA:Hexane, flow rate $0.5 \mathrm{~mL} \mathrm{~min}^{-}$ $\left.{ }^{1}, 211 \mathrm{~nm}, 30^{\circ} \mathrm{C}\right) \mathrm{t}_{\mathrm{R}}(2 S, 3 S): 8.8 \mathrm{~min}, \mathrm{t}_{\mathrm{R}}(2 R, 3 R): 9.5 \mathrm{~min}, 95 \%$ ee; $v_{\max }(\mathrm{KBr}) 2955(\mathrm{C}-\mathrm{H}), 1761(\mathrm{C}=\mathrm{O}), 1730(\mathrm{C}=\mathrm{O}), 1493$, $1155,1140(\mathrm{C}-\mathrm{F})$; Data for major diastereoisomer: $\delta_{\mathrm{H}}$ $\left(300 \mathrm{MHz}, \mathrm{CDCl}_{3}\right) 3.13(1 \mathrm{H}, \mathrm{dd}, J 18.2,3.7, \mathrm{C}(4) H \mathrm{H}), 3.32$ $(1 \mathrm{H}, \mathrm{dd}, J 18.3,9.1, \mathrm{C}(4) \mathrm{HH}), 3.69\left(3 \mathrm{H}, \mathrm{s}, \mathrm{CH}_{3}\right) 3.85(1 \mathrm{H}, \mathrm{d}$, $J$ 10.8, C(2)H), $3.93(1 \mathrm{H}, \mathrm{td}, J$ 9.8, 4.1, C(3)H), 6.98-7.17 
(9H, m, $\mathrm{Ar} H) ; \delta_{\mathrm{C}}\left(100 \mathrm{MHz}, \mathrm{CDCl}_{3}\right) 41.1(C(4)), 43.4(C(3))$, $52.6\left(C_{3}\right) 56.5(C(2)), 115.4\left(\mathrm{q}, J 290, C \mathrm{~F}_{3}\right) 127.4(\operatorname{ArC})$, $128.1(\mathrm{ArC}), 128.7$ ( $\mathrm{ArC}), 128.7$ ( $\mathrm{ArC}), 129.9(\mathrm{ArC}), 133.6$ (4ry $A r C), 134.8$ (4ry $A r C), 139.2 \quad(\mathrm{C}(3) \operatorname{ArC}(1)), 172.9$ $(C(1)=\mathrm{O}), 189.1(\mathrm{q}, J 35.5, C(5)=\mathrm{O}) ; \delta_{\mathrm{F}}\left(470 \mathrm{MHz}, \mathrm{CDCl}_{3}\right)-$ $79.5\left(\mathrm{CF}_{3}\right)$; Selected data for minor diastereoisomer: $\delta_{\mathrm{H}}$ $\left(300 \mathrm{MHz}, \mathrm{CDCl}_{3}\right) 2.70(1 \mathrm{H}, \mathrm{dd}, J 18.2,3.4, \mathrm{C}(4) H \mathrm{H}), 2.92-$ $3.02(1 \mathrm{H}, \mathrm{m}, \mathrm{C}(4) \mathrm{H} H) ; \delta_{\mathrm{C}}\left(100 \mathrm{MHz}, \mathrm{CDCl}_{3}\right) 40.3(C(4))$, $43.3(C(3)), 52.2\left(C_{3}\right), 56.9(C(2)), 127.8(\mathrm{ArC}), 128.0$ ( $A r C), 128.9(A r C), 129.5(A r C), 130.0(A r C), 133.4$ (4ry $A r C), 134.5$ (4ry $A r C), 139.9(\mathrm{C}(3) \operatorname{ArC}(1)), 172.0(C(1)=\mathrm{O})$; $\delta_{\mathrm{F}}\left(470 \mathrm{MHz}, \mathrm{CDCl}_{3}\right)-79.7\left(\mathrm{CF}_{3}\right) ; m / z\left(\mathrm{NSI}^{+}\right) 385\left([\mathrm{M}+\mathrm{H}]^{+}\right.$, $82 \%)$; HRMS ( $\left.\mathrm{NSI}^{+}\right) \quad \mathrm{C}_{19} \mathrm{H}_{17} \mathrm{ClF}_{3} \mathrm{O}_{3}^{+}\left([\mathrm{M}+\mathrm{H}]^{+}\right)$requires 385.0813; found 385.0818 (+1.3 ppm).

(2R,3R)-methyl 2-(4-bromophenyl)-6,6,6-trifluoro-5-oxo3-phenylhexanoate 9. Following general procedure $\mathrm{C}$, anhydride 54 (103 mg, $0.25 \mathrm{mmol}$ ), enone 2 (40.0 mg, 0.20 mmol), HBTM-2.1 (2S,3R)-3 (3.08 mg, $0.01 \mathrm{mmol}, 5 \mathrm{~mol} \%)$, DIPEA ( $43.5 \mu \mathrm{L}, 0.25 \mathrm{mmol})$ in $\mathrm{CH}_{2} \mathrm{Cl}_{2}(2 \mathrm{~mL})$ for $16 \mathrm{~h}$ at $78{ }^{\circ} \mathrm{C}$ followed by methanol $(2 \mathrm{~mL})$ gave $(2 R, 3 R)-9$ (90:10 dr). Chromatographic purification (10\% EtOAc:petrol eluent) gave pure product $(2 R, 3 R)-9(90: 10 \mathrm{dr})$ as a white solid (52 $\mathrm{mg}, 71 \%)$; mp $62-64{ }^{\circ} \mathrm{C} ;[\alpha]_{\mathrm{D}}{ }^{20}-118.5\left(c 0.15, \mathrm{CH}_{2} \mathrm{Cl}_{2}\right.$ ); Chiral HPLC chiralpak AD-H (5\% IPA:hexane, flow rate 1 $\left.\mathrm{ml} \mathrm{min}{ }^{-1}, 211 \mathrm{~nm}\right) \mathrm{t}_{\mathrm{R}}(2 S, 3 S) 5.6 \mathrm{~min}, \mathrm{t}_{\mathrm{R}}(2 R, 3 R) 6.8 \mathrm{~min}, 96 \%$ $e e ; v_{\max }(\mathrm{KBr}) 3065,3030,2956(\mathrm{C}-\mathrm{H}), 1762(\mathrm{C}=\mathrm{O}), 1734$ $(\mathrm{C}=\mathrm{O}), 1639,1618(\mathrm{C}=\mathrm{C})$; Data for major diastereoisomer: $\delta_{\mathrm{H}}$ (300 MHz, $\left.\mathrm{CDCl}_{3}\right)$ 3.09-3.16 (1H, m, C $\left.(4) H \mathrm{H}\right), 3.31(1 \mathrm{H}$, ddd, $J 18.3,9.2,0.5, \mathrm{C}(4) \mathrm{H} H), 3.69\left(3 \mathrm{H}, \mathrm{s}, \mathrm{CH}_{3}\right), 3.83(1 \mathrm{H}, \mathrm{d}$, $J$ 10.8, C(2)H), $3.92(1 \mathrm{H}, \mathrm{td}, J 10.4,3.9, \mathrm{C}(3) H), 6.96-7.00$ $(4 \mathrm{H}, \mathrm{m}, \operatorname{Ar} H), 7.07-7.18(3 \mathrm{H}, \mathrm{m}, \mathrm{Ar} H), 7.26-7.29(2 \mathrm{H}, \mathrm{m}$, $\mathrm{Ar} H) ; \delta_{\mathrm{C}}\left(100 \mathrm{MHz}, \mathrm{CDCl}_{3}\right) 41.1(C(4)), 43.4(C(3)), 52.6$ $\left(\mathrm{CH}_{3}\right), 56.5(\mathrm{C}(2)), 115.4$ (q, $\left.J 290, \mathrm{CF}_{3}\right), 121.7(\mathrm{C}(2) \operatorname{ArC}(4))$, $127.4(\mathrm{C}(3) \operatorname{ArC}(4)), 128.0(\operatorname{ArC}), 128.7(\operatorname{ArC}), 130.2(\operatorname{ArC})$, $131.7(\operatorname{ArC}), 135.3(\mathrm{C}(2) \operatorname{ArC}(1)), 139.2(\mathrm{C}(3) \operatorname{ArC}(1)), 172.8$ $(C(1)=\mathrm{O}), 189.0(\mathrm{q}, J 35.5, C(5)=\mathrm{O}) ; \delta_{\mathrm{F}}\left(282 \mathrm{MHz}, \mathrm{CDCl}_{3}\right)-$ $80.0\left(\mathrm{CF}_{3}\right)$; Selected data for minor diastereoisomer: $\delta_{\mathrm{H}}(300$ $\left.\mathrm{MHz}, \mathrm{CDCl}_{3}\right)$ 2.66-2.73 (1H, m, C(4)HH), $2.97(1 \mathrm{H}, \mathrm{ddd}, J$ $18.2,10.0,0.5, \mathrm{C}(4) \mathrm{H} H) ; \delta_{\mathrm{C}}\left(100 \mathrm{MHz}, \mathrm{CDCl}_{3}\right) 40.6(C(4))$, $43.2(C(3)), 52.3\left(C_{3}\right), 56.9(C(2)), 121.3(C(2) \operatorname{ArC}(4))$, 128.8 ( $A r C), 130.4(A r C), 131.1(\operatorname{ArC}), 131.8(A r C), 132.4$ $(\operatorname{ArC}), \quad 135.0 \quad(\mathrm{C}(2) \operatorname{ArC}(1)), \quad 139.9 \quad(\mathrm{C}(2) \operatorname{ArC}(1)), \quad 172.0$ $(C(1)=\mathrm{O}) ; \delta_{\mathrm{F}}\left(282 \mathrm{MHz}, \mathrm{CDCl}_{3}\right)-80.2\left(\mathrm{CF}_{3}\right) ; \mathrm{m} / z\left(\mathrm{NSI}^{+}\right) 429$ $\left([\mathrm{M}+\mathrm{H}]^{+}, 20 \%\right)$; HRMS $\left(\mathrm{NSI}^{+}\right) \mathrm{C}_{19} \mathrm{H}_{17}{ }^{79} \mathrm{BrF}_{3} \mathrm{O}_{3}{ }^{+}\left([\mathrm{M}+\mathrm{H}]^{+}\right)$ requires 429.0308; found 429.0311 (+0.8 ppm).

(2R,3R)-methyl 6,6,6-trifluoro-2-(4-methoxyphenyl)-5oxo-3-phenylhexanoate 10. Following general procedure $\mathrm{C}$, anhydride 56 ( $78.5 \mathrm{mg}, 0.25 \mathrm{mmol})$, enone $2(40.0 \mathrm{mg}, 0.20$ mmol), HBTM-2.1 (2S,3R)-3 (3.08 mg, $0.01 \mathrm{mmol}, 5 \mathrm{~mol} \%)$, DIPEA $(43.5 \mu \mathrm{L}, 0.25 \mathrm{mmol})$ in $\mathrm{CH}_{2} \mathrm{Cl}_{2}(2 \mathrm{~mL})$ for $16 \mathrm{~h}$ at $78{ }^{\circ} \mathrm{C}$ followed by methanol $(2 \mathrm{~mL})$ gave $(2 R, 3 R)-\mathbf{1 0}(97: 3$ dr). Chromatographic purification $\left(5 \% \mathrm{Et}_{2} \mathrm{O}\right.$ :petrol eluent) gave pure product $(2 R, 3 R)-\mathbf{1 0}(97: 3 \mathrm{dr})$ as a white solid (44.5 $\mathrm{mg}, 59 \%) ; \mathrm{mp} 86-90{ }^{\circ} \mathrm{C} ;[\alpha]_{\mathrm{D}}{ }^{20}-125.2\left(c 0.5, \mathrm{CH}_{2} \mathrm{Cl}_{2}\right)$; Chiral
HPLC Chiralpak IA (5\% IPA:Hexane, flow rate $1 \mathrm{~mL} \mathrm{~min}^{-1}$, $\left.211 \mathrm{~nm}, 30^{\circ} \mathrm{C}\right) \mathrm{t}_{\mathrm{R}}(2 S, 3 S): 5.7 \mathrm{~min}, \mathrm{t}_{\mathrm{R}}(2 R, 3 R): 6.5 \mathrm{~min}, 99 \%$ $e e ; v_{\max }(\mathrm{KBr}) 2949(\mathrm{C}-\mathrm{H}), 1761(\mathrm{C}=\mathrm{O}), 1730(\mathrm{C}=\mathrm{O}), 1514$, $1433,1254,1161,1136 \quad(\mathrm{C}-\mathrm{F}) ; \quad$ Data for major diastereoisomer: $\delta_{\mathrm{H}}\left(300 \mathrm{MHz}, \mathrm{CDCl}_{3}\right) 3.13(1 \mathrm{H}, \mathrm{dd}, J 18.2$, 3.8, C(4)HH), $3.33(1 \mathrm{H}, \mathrm{dd}, J 18.2,9.4, \mathrm{C}(4) \mathrm{H} H), 3.68(3 \mathrm{H}, \mathrm{s}$, $\left.\mathrm{CH}_{3}\right), 3.71\left(3 \mathrm{H}, \mathrm{s}, \mathrm{CH}_{3}\right) 3.80-3.95(2 \mathrm{H}, \mathrm{m}, \mathrm{C}(2) H$ and $\mathrm{C}(3) H)$, 6.67-6.70 (2H, m, C(2)Ar(3,5)H), 6.99-7.04 (4H, m, $\operatorname{Ar} H)$, 7.08-7.17 (3H, m, $\mathrm{Ar} H) ; \delta_{\mathrm{C}}\left(75 \mathrm{MHz}, \mathrm{CDCl}_{3}\right) 40.9(C(4))$, $43.6(\mathrm{C}(3)), 52.4\left(\mathrm{CH}_{3}\right) 55.4\left(\mathrm{CH}_{3}\right), 56.3(\mathrm{C}(2)), 114.0$ $(\mathrm{C}(2) \operatorname{ArC}(3,5)), 127.2 \quad(\mathrm{C}(3) \operatorname{ArC}(4)), 128.1 \quad(\operatorname{ArC}), 128.3$ (C(2)ArC(1)), $128.5(\operatorname{ArC}), 129.6(\operatorname{ArC}), 139.7$ (C(3)ArC(1)), $159.0 \quad(\mathrm{C}(2) \operatorname{ArC}(4)), 173.5 \quad(C(1)=\mathrm{O}), 189.3 \quad(\mathrm{q}, J$ 35.5, $C(5)=\mathrm{O}) ; \delta_{\mathrm{F}}\left(470 \mathrm{MHz}, \mathrm{CDCl}_{3}\right)-79.5\left(\mathrm{CF}_{3}\right)$; Selected data for minor diastereoisomer: $\delta_{\mathrm{H}}\left(300 \mathrm{MHz}, \mathrm{CDCl}_{3}\right) 2.72(1 \mathrm{H}, \mathrm{dd}, J$ 18.2, 3.5, C(4)HH), $2.95(1 \mathrm{H}, \mathrm{dd}, J 18.1,10.1, \mathrm{C}(4) \mathrm{H} H) ; \delta_{\mathrm{C}}$ (75MHz, $\left.\mathrm{CDCl}_{3}\right) 40.6(C(4)), 43.3(C(3)), 52.0\left(\mathrm{CH}_{3}\right), 55.5$ $\left(\mathrm{CH}_{3}\right), 56.8(C(2)), 114.7(\mathrm{C}(2) \operatorname{ArC}(3,5)), 128.1(\mathrm{ArC}), 128.8$ $(\operatorname{ArC}), 129.7(\operatorname{ArC}) ; \delta_{\mathrm{F}}\left(470 \mathrm{MHz}, \mathrm{CDCl}_{3}\right)-79.7\left(\mathrm{CF}_{3}\right) ; \mathrm{m} / \mathrm{z}$ $\left(\mathrm{NSI}^{+}\right) 381\left([\mathrm{M}+\mathrm{H}]^{+}, 75 \%\right) ; \mathrm{HRMS}\left(\mathrm{NSI}^{+}\right) \mathrm{C}_{20} \mathrm{H}_{20} \mathrm{~F}_{3} \mathrm{O}_{4}^{+}$ $\left([\mathrm{M}+\mathrm{H}]^{+}\right)$requires 381.1308 ; found $381.1312(+1.0 \mathrm{ppm})$.

\section{$(2 R, 3 R)$-methyl}

tolyl)hexanoate 11 . Following anhydride $\mathbf{5 3}(71.0 \mathrm{mg}, 0.25 \mathrm{mmol})$, enone $2(40.0 \mathrm{mg}, 0.20$ mmol), HBTM-2.1 (2S,3R)-3 (3.80 mg, $0.01 \mathrm{mmol}, 5 \mathrm{~mol} \%)$, DIPEA $(43.5 \mu \mathrm{L}, 0.25 \mathrm{mmol})$ in $\mathrm{CH}_{2} \mathrm{Cl}_{2}(2 \mathrm{~mL})$ for $16 \mathrm{~h}$ at $78{ }^{\circ} \mathrm{C}$ followed by methanol $(2 \mathrm{~mL})$ gave $(2 R, 3 R)-11(93: 7$ dr). Chromatographic purification $\left(5 \% \mathrm{Et}_{2} \mathrm{O}\right.$ :petrol eluent) gave pure product $(2 R, 3 R)-\mathbf{1 1}(93: 7 \mathrm{dr})$ as a white solid $(51.7$ $\mathrm{mg}, 71 \%$ ) with identical spectroscopic data as previously reported; ${ }^{11 \mathrm{e}}$ Chiral HPLC chiralpak AD-H (5\% IPA:hexane, flow rate $\left.1 \mathrm{ml} \mathrm{min}^{-1}, 211 \mathrm{~nm}\right) \mathrm{t}_{\mathrm{R}}(2 S, 3 S) 4.4 \mathrm{~min}, \mathrm{t}_{\mathrm{R}}(2 R, 3 R) 5.1$ $\min , 98 \% e e$.

(2R,3R)-methyl 6,6,6-trifluoro-2-(naphthalen-2-yl)-5-oxo3-phenylhexanoate 12. Following general procedure $C$, anhydride $57(88.5 \mathrm{mg}, 0.25 \mathrm{mmol})$, enone $2(40.0 \mathrm{mg}, 0.20$ mmol), HBTM-2.1 (2S,3R)-3 (3.08 mg, $0.01 \mathrm{mmol}, 5 \mathrm{~mol} \%)$, DIPEA $(43.5 \mu \mathrm{L}, 0.25 \mathrm{mmol})$ in $\mathrm{CH}_{2} \mathrm{Cl}_{2}(2 \mathrm{~mL})$ for $16 \mathrm{~h}$ at $78{ }^{\circ} \mathrm{C}$ followed by methanol $(2 \mathrm{~mL})$ gave crude product $(2 R, 3 R)-12(94: 6 \mathrm{dr})$. Chromatographic purification $(5 \%$ $\mathrm{Et}_{2} \mathrm{O}$ :petrol eluent) gave $(2 R, 3 R) \mathbf{- 1 2}(94: 6 \mathrm{dr})$ as a white solid $(57.1 \mathrm{mg}, 71 \%)$; mp $112-114{ }^{\circ} \mathrm{C} ;[\alpha]_{\mathrm{D}}{ }^{20}-183.7$ (c 0.3 , $\mathrm{CH}_{2} \mathrm{Cl}_{2}$ ); Chiral HPLC Chiralpak AS-H (1\% IPA:Hexane, flow rate $\left.1 \mathrm{~mL} \mathrm{~min}^{-1}, 220 \mathrm{~nm}, 30{ }^{\circ} \mathrm{C}\right) \mathrm{t}_{\mathrm{R}}(2 S, 3 S): 7.3 \mathrm{~min}$, $\mathrm{t}_{\mathrm{R}}(2 R, 3 R): 9.2 \mathrm{~min},>99 \%$ ee; $v_{\max }(\mathrm{KBr}) 2953,1759(\mathrm{C}=\mathrm{O})$, $1730(\mathrm{C}=\mathrm{O}), 1433,1283,1151,1136(\mathrm{C}-\mathrm{F})$; Data for major diastereoisomer: $\delta_{\mathrm{H}}\left(300 \mathrm{MHz}, \mathrm{CDCl}_{3}\right) 3.17-3.24(1 \mathrm{H}, \mathrm{m}$, $\mathrm{C}(4) H \mathrm{H}), 3.36-3.45(1 \mathrm{H}, \mathrm{m}, \mathrm{C}(4) \mathrm{H} H), 3.69\left(3 \mathrm{H}, \mathrm{s}, \mathrm{CH}_{3}\right)$, 4.04-4.14 $(2 \mathrm{H}, \mathrm{m}, \mathrm{C}(2) H$ and $\mathrm{C}(3) H), 7.01-7.12(5 \mathrm{H}, \mathrm{m}$, $\operatorname{Ar} H), 7.26(1 \mathrm{H}, \mathrm{dd}, J 8.5,1.9, \operatorname{Ar} H), 7.40-7.45(2 \mathrm{H}, \mathrm{m}, \operatorname{Ar} H)$, $7.58(1 \mathrm{H}, \mathrm{d}, J$ 1.7, $\operatorname{Ar} H), 7.65-7.74(3 \mathrm{H}, \mathrm{m}, \operatorname{Ar} H) ; \delta_{\mathrm{C}}$ $\left(100 \mathrm{MHz}, \mathrm{CDCl}_{3}\right) 41.0(C(4)), 43.4(C(3)), 52.5\left(\mathrm{CH}_{3}\right), 57.1$ $(C(2)), 115.3$ (q, $\left.J 290, C F_{3}\right), 126.2(A r C), 126.2(A r C), 126.3$ $(A r C), 127.2(A r C), 127.7(A r C), 127.8(A r C), 127.9(\operatorname{ArC})$, 
$128.1(\mathrm{ArC}), 128.3(\mathrm{ArC}), 128.6(\mathrm{ArC}), 132.7$ (4ry $\mathrm{ArC})$, 133.3 (4ry $A r C$ ), 133.7 (4ry $A r C$ ), 139.4 (C(3) $A r C(1)$ ), 173.2 $(C(1)=\mathrm{O}), 189.3(\mathrm{q}, J 35.4, C(5)=\mathrm{O}) ; \delta_{\mathrm{F}}\left(282 \mathrm{MHz}, \mathrm{CDCl}_{3}\right)-$ $80.0\left(\mathrm{CF}_{3}\right)$; Selected data for minor diastereoisomer: $\delta_{\mathrm{H}}$ $\left(300 \mathrm{MHz}, \mathrm{CDCl}_{3}\right)$ 2.68-2.75 (1H, m, C $\left.(4) H \mathrm{H}\right) .2 .97-3.06$ $(1 \mathrm{H}, \mathrm{m}, \mathrm{C}(4) \mathrm{H} H) ; \delta_{\mathrm{C}}\left(100 \mathrm{MHz}, \mathrm{CDCl}_{3}\right) 40.6(C(4)), 43.2$ $(C(3)), 52.4\left(\mathrm{CH}_{3}\right), 57.7(C(2)), 172.3(C(1)=\mathrm{O}) ; \delta_{\mathrm{F}}(282$ $\left.\mathrm{MHz}, \mathrm{CDCl}_{3}\right)-80.2\left(\mathrm{CF}_{3}\right) ; \mathrm{m} / z\left(\mathrm{NSI}^{+}\right) 418\left(\left[\mathrm{M}+\mathrm{NH}_{4}\right]^{+}, 65 \%\right)$; HRMS $\left(\mathrm{NSI}^{+}\right) \mathrm{C}_{23} \mathrm{H}_{23} \mathrm{~F}_{3} \mathrm{NO}_{3}{ }^{+}\left(\left[\mathrm{M}+\mathrm{NH}_{4}\right]^{+}\right)$requires 418.1625; found $418.1626(+0.3 \mathrm{ppm})$.

$(2 R, 3 R)$-methyl 6,6,6-trifluoro-5-oxo-3-phenyl-2-(thiophen3-yl)-hexanoate 13. Following general procedure $C$, anhydride $58(66.5 \mathrm{mg}, 0.25 \mathrm{mmol})$, enone 2 ( $40.0 \mathrm{mg}, 0.20 \mathrm{mmol})$, HBTM-2.1 $(2 S, 3 R)-3(3.08 \mathrm{mg}, 0.01 \mathrm{mmol}, 5 \mathrm{~mol} \%)$, DIPEA $(43.5 \mu \mathrm{L}, 0.25 \mathrm{mmol})$ in $\mathrm{CH}_{2} \mathrm{Cl}_{2}(2 \mathrm{~mL})$ for $16 \mathrm{~h}$ at $-78{ }^{\circ} \mathrm{C}$ followed by methanol $(2 \mathrm{~mL})$ gave crude product $(2 R, 3 R)-\mathbf{1 3}$ $(78: 22 \mathrm{dr})$. Chromatographic purification $\left(5 \% \mathrm{Et}_{2} \mathrm{O}\right.$ :petrol eluent) gave $(2 R, 3 R)-\mathbf{1 3}(78: 22 \mathrm{dr})$ as a white solid $(57.5 \mathrm{mg}$, $81 \%) ; \mathrm{mp} 82-85^{\circ} \mathrm{C} ;[\alpha]_{\mathrm{D}}{ }^{20}-54.0\left(c 0.3, \mathrm{CH}_{2} \mathrm{Cl}_{2}\right)$; Chiral HPLC Chiralpak AD-H (5\% IPA:Hexane, flow rate $1 \mathrm{~mL} \mathrm{~min}^{-1}$, $211 \mathrm{~nm}) \mathrm{t}_{\mathrm{R}}(2 S, 3 S): 5.2 \mathrm{~min}, \mathrm{t}_{\mathrm{R}}(2 R, 3 R): 5.9 \mathrm{~min},>99 \%$ ee; $v_{\max }(\mathrm{KBr}) 1761(\mathrm{C}=\mathrm{O}), 1730(\mathrm{C}=\mathrm{O}), 1595,1433,1287,1246$, 1194 (C-F); Data for major diastereoisomer: $\delta_{\mathrm{H}}(400 \mathrm{MHz}$, $\left.\mathrm{CDCl}_{3}\right) 3.13(1 \mathrm{H}, \mathrm{dd}, J 18.4,4.2, \mathrm{C}(4) H \mathrm{H}), 3.35(1 \mathrm{H}, \mathrm{dd}, J$ $18.4,9.5, \mathrm{C}(4) \mathrm{HH}), 3.70\left(3 \mathrm{H}, \mathrm{s}, \mathrm{CH}_{3}\right), 3.89(1 \mathrm{H}, \mathrm{td}, J$ 9.6, 4.2, $\mathrm{C}(3) H), 4.02-4.05(1 \mathrm{H}, \mathrm{m}, \mathrm{C}(2) H), 6.86(1 \mathrm{H}, \mathrm{dd}, J 5.0,1.2$, $\operatorname{Ar} H), 6.94(1 \mathrm{H}, \mathrm{dd}, J 2.9,1.1, \operatorname{Ar} H), 7.03-7.05(2 \mathrm{H}, \mathrm{m}, \operatorname{Ar} H)$, 7.13-7.21 (4H, m, $\mathrm{Ar} H) ; \delta_{\mathrm{C}}\left(75 \mathrm{MHz}, \mathrm{CDCl}_{3}\right) 40.3(C(4)), 43.6$ $(C(3)), 52.4\left(C_{3}\right), 52.6,(C(2)), 115.4\left(\mathrm{q}, J 290, C \mathrm{~F}_{3}\right), 123.3$ $(A r C), 125.8(A r C), 127.4(A r C), 127.4(A r C), 127.9(A r C)$, $128.6(\operatorname{ArC}), 136.2(\mathrm{C}(2) \operatorname{ArC}(1)), 139.7(\mathrm{C}(3) \operatorname{ArC}(1)), 172.9$ $(C(1)=\mathrm{O}), 189.3(\mathrm{q}, J 35.3, C(5)=\mathrm{O}) ; \delta_{\mathrm{F}}\left(470 \mathrm{MHz}, \mathrm{CDCl}_{3}\right)-$ $79.5\left(\mathrm{CF}_{3}\right)$; Selected data for minor diastereoisomer: $\delta_{\mathrm{H}}$ $\left(400 \mathrm{MHz}, \mathrm{CDCl}_{3}\right) 2.83(1 \mathrm{H}, \mathrm{dd}, J 18.3,3.8, \mathrm{C}(4) H \mathrm{H}), 2.99$ $(1 \mathrm{H}, \mathrm{dd}, J 18.4,9.5, \mathrm{C}(4) \mathrm{H} H) ; \delta_{\mathrm{C}}\left(75 \mathrm{MHz}, \mathrm{CDCl}_{3}\right) 40.5(C(4))$, $43.6(C(3)), 52.1\left(\mathrm{CH}_{3}\right), 53.0(C(2)), 124.0(\operatorname{ArC}), 127.0(\operatorname{ArC})$, 127.2 (ArC), $127.7(A r C), 128.0(A r C), 128.8(A r C), 136.4$ $(\mathrm{C}(2) \operatorname{ArC}(1)), 140.2(\mathrm{C}(3) \operatorname{ArC}(1)), 172.1 \quad(C(1)=\mathrm{O}) ; \delta_{\mathrm{F}}(470$ $\left.\mathrm{MHz}, \mathrm{CDCl}_{3}\right)-79.6\left(\mathrm{CF}_{3}\right) ; \mathrm{m} / z\left(\mathrm{APCI}^{+}\right) 374\left(\left[\mathrm{M}+\mathrm{NH}_{4}\right]^{+}, 73 \%\right)$; HRMS $\left(\mathrm{APCI}^{+}\right) \quad \mathrm{C}_{17} \mathrm{H}_{19} \mathrm{~F}_{3} \mathrm{NO}_{3} \mathrm{~S}^{+} \quad\left(\left[\mathrm{M}+\mathrm{NH}_{4}\right]^{+}\right)$requires 374.1032; found 374.1036 (+1.0 ppm).

\section{Preparation of products (Table 3 )}

(2R,3R)-methyl 6,6,6-trifluoro-5-oxo-2,3-diphenylhexanoate 4. Following general procedure $C$, anhydride $1(63.5 \mathrm{mg}, 0.25$ $\mathrm{mmol}$ ), enone 2 ( $40.0 \mathrm{mg}, 0.20 \mathrm{mmol})$, HBTM-2.1 (2S,3R)-3 (3.09 $\mathrm{mg}, 0.01 \mathrm{mmol}, 5 \mathrm{~mol} \%)$ and DIPEA $(43.5 \mu \mathrm{L}, 0.25$ $\mathrm{mmol})$ in $\mathrm{CH}_{2} \mathrm{Cl}_{2}(2 \mathrm{~mL})$ for $16 \mathrm{~h}$ at $-78{ }^{\circ} \mathrm{C}$ followed by methanol $(2 \mathrm{~mL})$ gave crude product $(2 R, 3 R)-5(90: 10 \mathrm{dr})$. Chromatographic purification (5\% $\mathrm{Et}_{2} \mathrm{O}$ :petrol eluent) gave $(2 R, 3 R)-5(90: 10 \mathrm{dr})$ as a white solid $(54.0 \mathrm{mg}, 78 \%) ; \mathrm{mp} 62-64$ ${ }^{\circ} \mathrm{C} ;[\alpha]_{\mathrm{D}}{ }^{20}-90.4\left(c 0.45, \mathrm{CH}_{2} \mathrm{Cl}_{2}\right)$; Chiral HPLC Chiralpak AD$\mathrm{H}\left(5 \%\right.$ IPA:hexane, flow rate $\left.1 \mathrm{ml} \mathrm{min}{ }^{-1}, 211 \mathrm{~nm}, 20{ }^{\circ} \mathrm{C}\right)$ $\mathrm{t}_{\mathrm{R}}(2 S, 3 S) 4.7 \mathrm{~min}, \mathrm{t}_{\mathrm{R}}(2 R, 3 R) 5.4 \mathrm{~min}, 99 \%$ ee; $v_{\max }(\mathrm{KBr}) 3030$, $2956(\mathrm{C}-\mathrm{H}), 1762(\mathrm{C}=\mathrm{O}), 1734(\mathrm{C}=\mathrm{O}), 1601(\mathrm{C}=\mathrm{C})$; Data for major diastereoisomer: $\delta_{\mathrm{H}}\left(300 \mathrm{MHz}, \mathrm{CDCl}_{3}\right), 3.16(1 \mathrm{H}, \mathrm{ddd}, J$ $18.3,3.8,0.4, \mathrm{C}(4) \mathrm{H} H), 3.36(1 \mathrm{H}$, ddd, $J 18.3,9.4,0.5$, $\mathrm{C}(4) H \mathrm{H}), 3.69\left(3 \mathrm{H}, \mathrm{s}, \mathrm{CH}_{3}\right), 3.87(1 \mathrm{H}, \mathrm{d}, J 10.5, \mathrm{C}(2) H), 3.96$ (1H, td, $J$ 10.1, 4.0, C(3)H), 6.99-7.03 (2H, m, ArH), 7.07-7.17 $(8 \mathrm{H}, \mathrm{m}, \mathrm{Ar} H) ; \delta_{\mathrm{C}}\left(100 \mathrm{MHz}, \mathrm{CDCl}_{3}\right) 40.9(C(4)), 43.6(C(3))$, $52.4\left(C_{3}\right), 57.1(C(2)), 115.4\left(\mathrm{q}, J 290, C \mathrm{~F}_{3}\right), 127.2(\operatorname{ArC})$,
$127.6(\mathrm{ArC}), 128.1(\mathrm{ArC}), 128.5(\mathrm{ArC}), 128.5(\mathrm{ArC}), 128.6$ $(\operatorname{ArC}), \quad 136.2 \quad(\mathrm{C}(2) \operatorname{ArC}(1)), \quad 139.6 \quad(\mathrm{C}(3) \operatorname{ArC}(1)), \quad 173.2$ $(C(1)=\mathrm{O}), 189.3(\mathrm{q}, J 35.5 C(5)=\mathrm{O}) ; \delta_{\mathrm{F}}\left(470 \mathrm{MHz}, \mathrm{CDCl}_{3}\right)-$ $79.5\left(\mathrm{CF}_{3}\right)$; Selected data for minor diastereoisomer: $\delta_{\mathrm{H}}(300$ $\left.\mathrm{MHz}, \mathrm{CDCl}_{3}\right), 2.07(1 \mathrm{H}$, ddd, $J 18.4,3.6,0.3, \mathrm{C}(4) \mathrm{H} H), 2.91-$ $3.01(1 \mathrm{H}, \mathrm{m}, \mathrm{C}(4) H \mathrm{H}) ; \delta_{\mathrm{C}}\left(100 \mathrm{MHz}, \mathrm{CDCl}_{3}\right) 40.6(C(4)), 43.6$ $(C(3)), 52.1\left(\mathrm{CH}_{3}\right), 57.6(C(2)), 128.7(\mathrm{ArC}), 128.8(\mathrm{ArC})$, $129.3(\operatorname{ArC}), 136.1(\mathrm{C}(2) \operatorname{ArC}(1)), 140.4$ (C(3)ArC(1)), 172.4 $(C(1)=\mathrm{O}) ; \delta_{\mathrm{F}}\left(470 \mathrm{MHz}, \mathrm{CDCl}_{3}\right)-79.7\left(\mathrm{CF}_{3}\right) ; \mathrm{m} / z\left(\mathrm{NSI}^{+}\right) 350$ $\left([\mathrm{M}+\mathrm{H}]^{+}, \quad 100 \%\right) ; \quad \mathrm{HRMS} \quad\left(\mathrm{NSI}^{+}\right) \quad \mathrm{C}_{19} \mathrm{H}_{18} \mathrm{O}_{3} \mathrm{~F}_{3}^{+} \quad\left([\mathrm{M}+\mathrm{H}]^{+}\right)$ requires 351.1203; found $351.1206(+1.0 \mathrm{ppm})$.

(2R,3R)-ethyl 6,6,6-trifluoro-5-oxo-2,3-diphenylhexanoate 14. Following general procedure $\mathrm{C}$, anhydride $1(63.5 \mathrm{mg}, 0.25$ mmol), enone 2 (40.0 mg, $0.20 \mathrm{mmol})$, HBTM-2.1 (2S,3R)-3 (3.08 $\mathrm{mg}, 0.01 \mathrm{mmol}, 5 \mathrm{~mol} \%)$ and DIPEA (43.5 $\mu \mathrm{L}, 0.25$ $\mathrm{mmol})$ in $\mathrm{CH}_{2} \mathrm{Cl}_{2}(2 \mathrm{~mL})$ for $16 \mathrm{~h}$ at $-78{ }^{\circ} \mathrm{C}$ followed by ethanol (2 $\mathrm{mL})$ gave crude product $(2 R, 3 R)-14 \quad(85: 15 \mathrm{dr})$. Chromatographic purification (5\% $\mathrm{Et}_{2} \mathrm{O}$ :petrol eluent) gave $(2 R, 3 R)-14(85: 15 \mathrm{dr})$ as a white solid $(60.9 \mathrm{mg}, 84 \%)$; $\mathrm{mp} 60-$ $62^{\circ} \mathrm{C} ;[\alpha]_{\mathrm{D}}{ }^{20}-77.2\left(c 0.5, \mathrm{CH}_{2} \mathrm{Cl}_{2}\right)$; Chiral HPLC Chiralpak OJ-

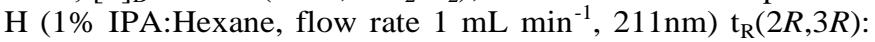
$6.9 \min , \mathrm{t}_{\mathrm{R}}(2 S, 3 S): 10.2 \mathrm{~min}, 99 \%$ ee; $v_{\max }(\mathrm{KBr}) 2943(\mathrm{C}-\mathrm{H})$, $1761(\mathrm{C}=\mathrm{O}), 1724(\mathrm{C}=\mathrm{O}), 1454,1136(\mathrm{C}-\mathrm{F})$; Data for major diastereoisomer: $\delta_{\mathrm{H}}\left(400 \mathrm{MHz}, \mathrm{CDCl}_{3}\right) 1.21\left(3 \mathrm{H}, \mathrm{t}, J \mathrm{~J} .1, \mathrm{CH}_{3}\right)$, $3.17(1 \mathrm{H}, \mathrm{dd}, J 18.3,3.7, \mathrm{C}(4) H \mathrm{H}), 3.38(1 \mathrm{H}, \mathrm{dd}, J 18.1,9.8$, $\mathrm{C}(4) \mathrm{H} H), 3.86(1 \mathrm{H}, \mathrm{d}, J 10.5, \mathrm{C}(2) H), 3.96(1 \mathrm{H}, \mathrm{td}, J$ 10.2, 3.7, $\mathrm{C}(3) \mathrm{H}), 4.08-4.23\left(2 \mathrm{H}, \mathrm{m}, \mathrm{CH}_{2} \mathrm{CH}_{3}\right), 7.02-7.04(2 \mathrm{H}, \mathrm{m}, \mathrm{ArH})$, 7.06-7.18 (8H, m, $\mathrm{ArH}) ; \delta_{\mathrm{C}}\left(100 \mathrm{MHz}, \mathrm{CDCl}_{3}\right) 14.1\left(\mathrm{CH}_{3}\right)$, $40.8(C(4)), 43.6(C(3)), 57.8(C(2)), 61.4\left(C_{2} \mathrm{CH}_{3}\right), 115.4$ (q, $J$ 290, $\left.C \mathrm{~F}_{3}\right), 127.2(\mathrm{ArC}), 127.6$ (ArC), 128.1 (ArC), 128.5 $(A r C), 128.5$ (ArC), 128.5 (ArC), 136.4 (C(2)ArC(1)), 139.7 $(\mathrm{C}(3) \operatorname{ArC}(1)), 172.7(C(1)=\mathrm{O}), 189.3(\mathrm{q}, J 35.4, C(5)=\mathrm{O}) ; \delta_{\mathrm{F}}$ (470 MHz, $\left.\mathrm{CDCl}_{3}\right)$-79.5 $\left(\mathrm{CF}_{3}\right)$; Selected data for minor diastereoisomer: $\delta_{\mathrm{H}}\left(400 \mathrm{MHz}, \mathrm{CDCl}_{3}\right) 0.91\left(3 \mathrm{H}, \mathrm{t}, J 7.1, \mathrm{CH}_{3}\right)$, $2.70(1 \mathrm{H}, \mathrm{dd}, J 18.3,3.5, \mathrm{C}(4) H \mathrm{H}), 2.97(1 \mathrm{H}, \mathrm{dd}, J 18.2,10.2$, $\mathrm{C}(4) \mathrm{H} H) ; \delta_{\mathrm{C}}\left(100 \mathrm{MHz}, \mathrm{CDCl}_{3}\right) 13.9\left(\mathrm{CH}_{3}\right), 40.7(C(4)), 43.4$ $(C(3)), 57.8(C(2)), 60.9\left(\mathrm{CH}_{2} \mathrm{CH}_{3}\right), 127.6(\mathrm{ArC}), 128.2(\mathrm{ArC})$, 128.7 ( $A r C$ ), 128.7 (ArC), 129.2 (ArC), 136.2 (C(2)ArC(1)), $140.4(\mathrm{C}(3) \operatorname{ArC}(1)), 171.8(C(1)=\mathrm{O}) ; \delta_{\mathrm{F}}\left(470 \mathrm{MHz}, \mathrm{CDCl}_{3}\right)-$ $79.7\left(\mathrm{CF}_{3}\right) ; \mathrm{m} / \mathrm{z}\left(\mathrm{NSI}^{+}\right) 365\left([\mathrm{M}+\mathrm{H}]^{+}, 95 \%\right)$; HRMS $\left(\mathrm{NSI}^{+}\right)$ $\mathrm{C}_{20} \mathrm{H}_{20} \mathrm{~F}_{3} \mathrm{O}_{3}{ }^{+}\left([\mathrm{M}+\mathrm{H}]^{+}\right)$requires 365.1359 ; found 365.1364 (+1.4 ppm).

(2R,3R)-allyl 6,6,6-trifluoro-5-oxo-2,3-diphenylhexanoate 15. Following general procedure $C$, anhydride $\mathbf{1}(63.5 \mathrm{mg}, 0.25$ $\mathrm{mmol}$ ), enone 2 (40.0 mg, $0.20 \mathrm{mmol})$, HBTM-2.1 (2S,3R)-3 (3.08 $\mathrm{mg}, 0.01 \mathrm{mmol}, 5 \mathrm{~mol} \%)$ and DIPEA (43.5 $\mu \mathrm{L}, 0.25$ $\mathrm{mmol})$ in $\mathrm{CH}_{2} \mathrm{Cl}_{2}(2 \mathrm{~mL})$ for $16 \mathrm{~h}$ at $-78{ }^{\circ} \mathrm{C}$ followed by allyl alcohol $(2 \mathrm{~mL})$ gave crude product $(2 R, 3 R)-15(88: 12 \mathrm{dr})$. Chromatographic purification (5\% $\mathrm{Et}_{2} \mathrm{O}$ :petrol eluent) gave $(2 R, 3 R)-15(88: 12 \mathrm{dr})$ as a white solid $(30.8 \mathrm{mg}, 42 \%)$; $\mathrm{mp} 44-$ $46{ }^{\circ} \mathrm{C} ;[\alpha]_{\mathrm{D}}{ }^{20}-85.6\left(c 0.5, \mathrm{CH}_{2} \mathrm{Cl}_{2}\right)$; Chiral HPLC Chiralpak AD-H (5\% IPA:Hexane, flow rate $\left.0.5 \mathrm{~mL} \mathrm{~min}^{-1}, 211 \mathrm{~nm}\right)$ $\mathrm{t}_{\mathrm{R}}(2 S, 3 S): 10.1 \mathrm{~min}, \mathrm{t}_{\mathrm{R}}(2 R, 3 R): 11.0 \mathrm{~min}, 97 \%$ ee; $v_{\max }(\mathrm{KBr})$ 3444, 3032, 2943 (C-H), 1767 (C=O), 1733 (C=O), 1456, 1152 (C-F); Data for major diastereoisomer: $\delta_{\mathrm{H}}\left(400 \mathrm{MHz}, \mathrm{CDCl}_{3}\right)$ $3.16(1 \mathrm{H}, \mathrm{dd}, J 18.3,3.5, \mathrm{C}(4) H \mathrm{H}), 3.33-3.40(1 \mathrm{H}, \mathrm{m}, \mathrm{C}(4) \mathrm{H} H)$, $3.89(1 \mathrm{H}, \mathrm{d}, J 10.5, \mathrm{C}(2) H), 3.93-3.97(1 \mathrm{H}, \mathrm{m}, \mathrm{C}(3) H), 4.54-$ $4.65(2 \mathrm{H}, \mathrm{m}$, allyl $\mathrm{CH} H$ and allyl $\mathrm{CHH}), 5.17-5.23(2 \mathrm{H}, \mathrm{m}$, $\mathrm{CH}=\mathrm{CH} H$ and $\mathrm{CH}=\mathrm{CHH}), 5.79-5.88\left(1 \mathrm{H}, \mathrm{m}, \mathrm{CH}=\mathrm{CH}_{2}\right), 7.01-$ $7.03(2 \mathrm{H}, \mathrm{m}, \mathrm{ArH}), 7.06-7.18(8 \mathrm{H}, \mathrm{m}, \mathrm{ArH}) ; \delta_{\mathrm{C}}(75 \mathrm{MHz}$, $\left.\mathrm{CDCl}_{3}\right) 40.8(C(4)), 43.6(C(3)), 57.2(C(2)), 65.9$ (allyl $\left.C \mathrm{H}_{2}\right)$, $118.9\left(\mathrm{CH}=\mathrm{CH}_{2}\right), 127.2(\mathrm{ArC}), 127.7$ (ArC), 128.1 (ArC), 128.5 
$(A r C), 128.6(A r C), 128.6(A r C), 131.7 \quad\left(C \mathrm{H}=\mathrm{CH}_{2}\right), 136.2$ $(\mathrm{C}(2) \operatorname{ArC}(1)), 139.6(\mathrm{C}(3) \operatorname{ArC}(1)), 172.4(C(1)=\mathrm{O}), 189.2$ (q, $J$ 35.3, $C(5)=\mathrm{O}) ; \delta_{\mathrm{F}}\left(282 \mathrm{MHz}, \mathrm{CDCl}_{3}\right)-80.0\left(\mathrm{CF}_{3}\right)$; Selected data for minor diastereoisomer: $\delta_{\mathrm{H}}\left(400 \mathrm{MHz}, \mathrm{CDCl}_{3}\right) 2.70(1 \mathrm{H}$, dd, $J$ 18.2, 3.4, C(4)HH), 2.93-3.00 (1H, m, C $(4) \mathrm{H} H), 4.22-4.36$ $(2 \mathrm{H}, \mathrm{m}$, allyl $\mathrm{CH} H$ and allyl $\mathrm{CHH}), 4.90-5.02(2 \mathrm{H}, \mathrm{m}$, $\mathrm{CH}=\mathrm{CH} H$ and $\mathrm{CH}=\mathrm{CHH}), 5.52(1 \mathrm{H}, \mathrm{ddt}, J 17.2,10.5,5.6$, $\left.\mathrm{CH}=\mathrm{CH}_{2}\right) ; \delta_{\mathrm{C}}\left(75 \mathrm{MHz}, \mathrm{CDCl}_{3}\right) 40.6(C(4)), 43.4(C(3)), 57.8$ $(C(2)), 65.5$ (Allyl $\left.\mathrm{CH}_{2}\right), 118.1\left(\mathrm{CH}=\mathrm{CH}_{2}\right), 128.2(\mathrm{ArC}), 128.7$ (ArC), 128.8 (ArC), 129.3 (ArC), 140.3 (C(3)ArC(1)), 171.6 $(C(1)=\mathrm{O}) ; \delta_{\mathrm{F}}\left(282 \mathrm{MHz}, \mathrm{CDCl}_{3}\right)-80.2\left(\mathrm{CF}_{3}\right) ; \mathrm{m} / z\left(\mathrm{NSI}^{+}\right) 377$ $\left([\mathrm{M}+\mathrm{H}]^{+}, 75 \%\right)$; HRMS $\left(\mathrm{NSI}^{+}\right) \mathrm{C}_{21} \mathrm{H}_{20} \mathrm{~F}_{3} \mathrm{O}_{3}{ }^{+}\left([\mathrm{M}+\mathrm{H}]^{+}\right)$requires 377.1359 ; found 377.1363 (+1.0 ppm).

(3R,4R)-4-(4-chlorophenyl)-3-phenyl-6-(trifluoromethyl)3,4-dihydro-2H-pyran-2-one 16. Following general procedure $\mathrm{B}$, anhydride $\mathrm{I}(63.5 \mathrm{mg}, 0.25 \mathrm{mmol})$ in $\mathrm{CH}_{2} \mathrm{Cl}_{2}(2 \mathrm{~mL})$, HBTM-2.1 (2S,3R)-3 (3.09 mg, $0.01 \mathrm{mmol}, 5 \mathrm{~mol} \%)$, enone 36 (46.9 mg, $0.20 \mathrm{mmol})$ and DIPEA $(43.5 \mu \mathrm{L}, 0.25 \mathrm{mmol})$ for 16 $\mathrm{h}$ at $-78{ }^{\circ} \mathrm{C}$ gave crude lactone $(3 R, 4 R)-16(85: 15 \mathrm{dr})$. Chromatographic purification (eluent $\mathrm{Et}_{2} \mathrm{O}$ :petrol 5:95) gave lactone $(3 R, 4 R)-16(>99: 1 \mathrm{dr})$ as a white solid $(59.8 \mathrm{mg}, 85 \%)$ with identical spectroscopic data as previously reported; ${ }^{11 \mathrm{e}}$ Chiral HPLC Chiralpak AD-H (2\% IPA:hexane, flow rate $2 \mathrm{~mL}$ $\left.\min ^{-1}, 211 \mathrm{~nm}, 20^{\circ} \mathrm{C}\right) \mathrm{t}_{\mathrm{R}}(3 R, 4 R): 10.2 \mathrm{~min}, \mathrm{t}_{\mathrm{R}}(3 S, 4 S): 30.3 \mathrm{~min}$, $>99 \%$ ee .

(2R,3R)-methyl 6,6,6-trifluoro-3-(4-methoxyphenyl)-5-oxo2-phenylhexanoate 17. Following general procedure $C$, anhydride 1 (63.5 mg, $0.25 \mathrm{mmol}$ ), enone 40 (46.0 $\mathrm{mg}, 0.20$ mmol), HBTM-2.1 (2S,3R)-3 (3.08 mg, $0.01 \mathrm{mmol}, 5 \mathrm{~mol} \%)$ and DIPEA $(43.5 \mu \mathrm{L}, 0.25 \mathrm{mmol})$ for $16 \mathrm{~h}$ at $-78{ }^{\circ} \mathrm{C}$ in $\mathrm{CH}_{2} \mathrm{Cl}_{2}$ $(2 \mathrm{~mL})$, followed by methanol $(2 \mathrm{~mL})$ gave crude product $(2 R, 3 R)-17(95: 5 \mathrm{dr})$. Chromatographic purification (5\% $\mathrm{Et}_{2} \mathrm{O}$ :petrol eluent) gave $(2 R, 3 R)-\mathbf{1 7}(95: 5 \mathrm{dr})$ as a colourless oil $(70.5 \mathrm{mg}, 93 \%) ;[\alpha]_{\mathrm{D}}{ }^{20}-38.7\left(c 0.6, \mathrm{CH}_{2} \mathrm{Cl}_{2}\right)$; Chiral HPLC

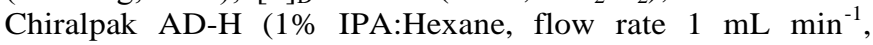
$211 \mathrm{~nm}) \mathrm{t}_{\mathrm{R}}(2 S, 3 S): 12.0 \mathrm{~min}, \mathrm{t}_{\mathrm{R}}(2 R, 3 R): 16.4 \mathrm{~min}, 98 \%$ ee; $v_{\max }$, $(\mathrm{KBr}) 2955(\mathrm{C}-\mathrm{H}), 1765(\mathrm{C}=\mathrm{O}), 1730(\mathrm{C}=\mathrm{O}), 1514,1144(\mathrm{C}-$ F), 829; Data for major diastereoisomer: $\delta_{\mathrm{H}}\left(500 \mathrm{MHz}, \mathrm{CDCl}_{3}\right)$ $3.12(1 \mathrm{H}, \mathrm{dd}, J 18.2,3.7, \mathrm{C}(4) H \mathrm{H}), 3.32(1 \mathrm{H}, \mathrm{dd}, J 18.2,9.8$, $\mathrm{C}(4) \mathrm{H} H), 3.68(3 \mathrm{H}, \mathrm{s} \mathrm{CH}), 3.69(3 \mathrm{H}, \mathrm{s} \mathrm{CH}), 3.84(1 \mathrm{H}, \mathrm{d}, J$ 10.4, C(2)H), $3.92(1 \mathrm{H}, \mathrm{td}, J 10.1,3.7, \mathrm{C}(3) H), 6.65-6.67(2 \mathrm{H}$, m, C(3) $\operatorname{Ar}(3,5) H), 6.92-6.94(2 \mathrm{H}, \mathrm{m}, \mathrm{C}(3) \operatorname{Ar}(2,6) H), 7.11-7.19$ $(5 \mathrm{H}, \mathrm{m}, \mathrm{Ar} H) ; \delta_{\mathrm{C}}\left(125 \mathrm{MHz}, \mathrm{CDCl}_{3}\right) 41.0(C(4)), 42.8(C(3))$, $52.4\left(\mathrm{CH}_{3}\right), 55.2\left(\mathrm{CH}_{3}\right), 57.2(\mathrm{C}(2)), 113.8(\mathrm{C}(3) \mathrm{ArC}(3,5))$, 115.4 (q, $\left.J 291, C F_{3}\right), 127.6(\mathrm{C}(2) A r C(4)), 128.5(A r C), 128.6$ $(A r C), 129.1(\operatorname{ArC}), 131.5(\mathrm{C}(3) \operatorname{ArC}(1)), 136.3(\mathrm{C}(2) \operatorname{ArC}(1))$, $158.5(\mathrm{C}(3) A r C(4)), 173.3(C(1)=\mathrm{O}), 189.3(\mathrm{q}, J 35.1, C(5)=\mathrm{O})$; $\delta_{\mathrm{F}}\left(470 \mathrm{MHz}, \mathrm{CDCl}_{3}\right)-79.5\left(\mathrm{CF}_{3}\right)$; Selected data for minor diastereoisomer: $\delta_{\mathrm{H}}\left(500 \mathrm{MHz}, \mathrm{CDCl}_{3}\right) 2.65-2.69(1 \mathrm{H}, \mathrm{m}$, $\mathrm{C}(4) H \mathrm{H}), 2.89-2.95(1 \mathrm{H}, \mathrm{m}, \mathrm{C}(4) \mathrm{H} H), 6.85(2 \mathrm{H}, \mathrm{d}, J 8.7$, $\mathrm{C}(3) \operatorname{Ar}(3,5) H) ; \delta_{\mathrm{C}}\left(125 \mathrm{MHz}, \mathrm{CDCl}_{3}\right) 40.7(C(4)), 42.5$ (C(3)), $52.1\left(\mathrm{CH}_{3}\right), 55.2\left(\mathrm{CH}_{3}\right), 57.8(C(2)), 128.6(\mathrm{ArC}), 129.3(\mathrm{ArC})$, $129.5(\mathrm{ArC}) ; \delta_{\mathrm{F}}\left(470 \mathrm{MHz}, \mathrm{CDCl}_{3}\right)-79.7\left(\mathrm{CF}_{3}\right) ; \mathrm{m} / z\left(\mathrm{NSI}^{+}\right) 381$ $\left([\mathrm{M}+\mathrm{H}]^{+}, 25 \%\right)$; HRMS $\left(\mathrm{NSI}^{+}\right) \mathrm{C}_{20} \mathrm{H}_{20} \mathrm{~F}_{3} \mathrm{O}_{4}{ }^{+}\left([\mathrm{M}+\mathrm{H}]^{+}\right)$requires 381.1308 ; found $381.1314(+1.5 \mathrm{ppm})$.

$(2 R, 3 R)$-methyl $\quad 6,6,6$-trifluoro-5-oxo-2-phenyl-3-( $p$ tolyl)hexanoate 18. Following general procedure $C$, anhydride 1 (63.5 mg, $0.25 \mathrm{mmol})$, enone 38 (43.0 $\mathrm{mg}, 0.20 \mathrm{mmol}$ ), HBTM-2.1 (2S,3R)-3 (3.80 mg, $0.01 \mathrm{mmol}, 5 \mathrm{~mol} \%)$, DIPEA $(43.5 \mu \mathrm{L}, 0.25 \mathrm{mmol})$ in $\mathrm{CH}_{2} \mathrm{Cl}_{2}(2 \mathrm{~mL})$ for $16 \mathrm{~h}$ at $-78{ }^{\circ} \mathrm{C}$ followed by methanol $(2 \mathrm{~mL})$ gave crude product $(2 R, 3 R)-\mathbf{1 8}$
(93:7 dr). Chromatographic purification (10\% EtOAc:petrol eluent) gave $(2 R, 3 R)-\mathbf{1 8}(93: 7 \mathrm{dr})$ as a colourless oil $(41.0 \mathrm{mg}$, $56 \%) ;[\alpha]_{\mathrm{D}}{ }^{20}-105.6\left(c 0.10, \mathrm{CH}_{2} \mathrm{Cl}_{2}\right)$; Chiral HPLC chiralpak AD-H (5\% IPA:hexane, flow rate $\left.1 \mathrm{ml} \mathrm{min}^{-1}, 211 \mathrm{~nm}\right) \mathrm{t}_{\mathrm{R}}(2 S, 3 S)$ $4.9 \mathrm{~min}, \mathrm{t}_{\mathrm{R}}(2 R, 3 R) 5.3 \mathrm{~min}, 98 \%$ ee; $v_{\max }(\mathrm{KBr}) 3030,2952(\mathrm{C}-$ $\mathrm{H}), 1760(\mathrm{C}=\mathrm{O}), 1723(\mathrm{C}=\mathrm{O}), 1616(\mathrm{C}=\mathrm{C})$; Data for major diastereoisomer: $\delta_{\mathrm{H}}\left(300 \mathrm{MHz}, \mathrm{CDCl}_{3}\right) 2.21\left(3 \mathrm{H}, \mathrm{s}, \mathrm{ArCH}_{3}\right)$, $3.14(1 \mathrm{H}$, dd, $J 18.3,3.4, \mathrm{C}(4) H \mathrm{H}), 3.35(1 \mathrm{H}, \mathrm{dd}, J 18.2,9.2$, $\mathrm{C}(4) \mathrm{H} H), 3.68\left(3 \mathrm{H}, \mathrm{s}, \mathrm{OCH}_{3}\right), 3.88(1 \mathrm{H}, \mathrm{d}, J 10.3, \mathrm{C}(2) H), 3.94$ $(1 \mathrm{H}, \mathrm{td}, J 9.8,3.8, \mathrm{C}(3) H), 6.89-6.96(4 \mathrm{H}, \mathrm{m}, \mathrm{Ar} H), 7.11-7.20$ $(5 \mathrm{H}, \mathrm{m}, \mathrm{Ar} H) ; \delta_{\mathrm{C}}\left(100 \mathrm{MHz}, \mathrm{CDCl}_{3}\right) 21.1\left(\mathrm{ArCH}_{3}\right), 40.9$ $(C(4)), 43.0(C(3)), 52.4\left(\mathrm{OCH}_{3}\right), 57.1(C(2)), 115.4(\mathrm{q}, J 290$, $\mathrm{CF}_{3}$ ), 127.6 ( $\mathrm{ArC}$ ), $127.9(\mathrm{ArC}), 128.5$ ( $\left.\mathrm{ArC}\right), 128.6(\mathrm{ArC})$, 129.2 ( $A r C$ ), 136.4 (4ry $A r C$ ), 136.5 (4ry $A r C$ ), 136.7 (4ry $A r C), 173.3(C(1)=\mathrm{O}), 189.3(\mathrm{q}, J 35.2, C(5)=\mathrm{O}) ; \delta_{\mathrm{F}}(470 \mathrm{MHz}$, $\left.\mathrm{CDCl}_{3}\right)$-79.5 $\left(\mathrm{CF}_{3}\right)$; Selected data for minor diastereoisomer: $\delta_{\mathrm{H}}\left(300 \mathrm{MHz}, \mathrm{CDCl}_{3}\right) 2.32\left(3 \mathrm{H}, \mathrm{s}, \mathrm{ArCH}_{3}\right), 2.69(1 \mathrm{H}, \mathrm{dd}, J$ $18.1,3.5, \mathrm{C}(4) H \mathrm{H}), 2.90-2.99(1 \mathrm{H}, \mathrm{m}, \mathrm{C}(4) \mathrm{H} H) ; \delta_{\mathrm{C}}(100 \mathrm{MHz}$, $\left.\mathrm{CDCl}_{3}\right) 21.2\left(\mathrm{ArCH}_{3}\right), 40.6(C(4)), 42.8(C(3)), 52.2\left(\mathrm{OCH}_{3}\right)$, $57.7(C(2)), 128.6(\mathrm{ArC}), 129.3(\mathrm{ArC}), 129.4(\mathrm{ArC}), 129.5$ $(\mathrm{ArC}) ; \delta_{\mathrm{F}}\left(470 \mathrm{MHz}, \mathrm{CDCl}_{3}\right)-79.7\left(\mathrm{CF}_{3}\right) ; \mathrm{m} / \mathrm{z}\left(\mathrm{NSI}^{+}\right) 365$ $\left([\mathrm{M}+\mathrm{H}]^{+}, 30 \%\right)$; HRMS $\left(\mathrm{NSI}^{+}\right) \mathrm{C}_{20} \mathrm{H}_{20} \mathrm{~F}_{3} \mathrm{O}_{3}{ }^{+}\left([\mathrm{M}+\mathrm{H}]^{+}\right)$requires 365.1359 ; found 365.1367 (+2.2 ppm).

$(3 R, 4 R)-4-($ naphthalen-2-yl)-3-phenyl-6-(trifluoromethyl)3,4-dihydro-2H-pyran-2-one 19. Following general procedure $\mathrm{B}$, anhydride $1(63.5 \mathrm{mg}, 0.25 \mathrm{mmol})$ in $\mathrm{CH}_{2} \mathrm{Cl}_{2}(2 \mathrm{~mL})$, HBTM-2.1 (2S,3R)-3 (3.09 mg, $0.01 \mathrm{mmol}, 5 \mathrm{~mol} \%)$, enone 37 (50.0 mg, $0.20 \mathrm{mmol})$ and DIPEA $(43.5 \mu \mathrm{L}, 0.25 \mathrm{mmol})$ for 16 $\mathrm{h}$ at $-78{ }^{\circ} \mathrm{C}$ gave crude lactone $(3 R, 4 R)-19(89: 11 \mathrm{dr})$. Chromatographic purification (eluent $\mathrm{Et}_{2} \mathrm{O}$ :petrol 5:95) gave lactone $(3 R, 4 R)-19(>99: 1 \mathrm{dr})$ as a white solid $(63.0 \mathrm{mg}, 86 \%)$ with identical spectroscopic data as previously reported; ${ }^{11 \mathrm{e}}$ Chiral HPLC Chiralpak AD-H (2\% IPA:hexane, flow rate $2 \mathrm{~mL}$ $\left.\min ^{-1}, 211 \mathrm{~nm}, 20^{\circ} \mathrm{C}\right) \mathrm{t}_{\mathrm{R}}(3 R, 4 R): 11.1 \mathrm{~min}, \mathrm{t}_{\mathrm{R}}(3 S, 4 S): 26.8 \mathrm{~min}$, $99 \%$ ee.

\section{$(2 R, 3 R)$-methyl}

6,6,6-trifluoro-3-(furan-2-yl)-5-oxo-2phenylhexanoate 20. Following general procedure C, anhydride 1 (63.5 mg, $0.25 \mathrm{mmol})$, enone 41 (38.0 mg, 0.20 mmol), HBTM-2.1 (2S,3R)-3 (3.08 mg, $0.01 \mathrm{mmol}, 5 \mathrm{~mol} \%)$ and DIPEA $(43.5 \mu \mathrm{L}, 0.25 \mathrm{mmol})$ in $\mathrm{CH}_{2} \mathrm{Cl}_{2}(2 \mathrm{~mL})$ for $16 \mathrm{~h}$ at $-78{ }^{\circ} \mathrm{C}$ followed by methanol $(2 \mathrm{~mL})$ gave crude product $(2 R, 3 R)-20(88: 12 \mathrm{dr})$. Chromatographic purification (5\% $\mathrm{Et}_{2} \mathrm{O}$ :petrol eluent) gave $(2 R, 3 R)-\mathbf{2 0}(88: 12 \mathrm{dr})$ as a white solid $(55.8 \mathrm{mg}, 81 \%)$; mp $48-50^{\circ} \mathrm{C} ;[\alpha]_{\mathrm{D}}{ }^{20}-89.8\left(c 0.57, \mathrm{CH}_{2} \mathrm{Cl}_{2}\right)$; Chiral HPLC Chiralpak OJ-H (1\% IPA:Hexane, flow rate $1 \mathrm{~mL}$ $\left.\min ^{-1}, 211 \mathrm{~nm}\right) \mathrm{t}_{\mathrm{R}}(2 R, 3 R): 7.6 \mathrm{~min}, \mathrm{t}_{\mathrm{R}}(2 S, 3 S): 9.8 \mathrm{~min},>99 \%$ ee; $v_{\max }(\mathrm{KBr}) 2957(\mathrm{C}-\mathrm{H}), 1761(\mathrm{C}=\mathrm{O}), 1728(\mathrm{C}=\mathrm{O}), 1140(\mathrm{C}-\mathrm{F})$, 1011; Data for major diastereoisomer: $\delta_{\mathrm{H}}\left(300 \mathrm{MHz}, \mathrm{CDCl}_{3}\right)$ 2.99-3.06 $(1 \mathrm{H}, \mathrm{m}, \mathrm{C}(4) H \mathrm{H}), 3.32-3.41(1 \mathrm{H}, \mathrm{m}, \mathrm{C}(4) \mathrm{H} H), 3.69$ $\left(3 \mathrm{H}, \mathrm{s}, \mathrm{CH}_{3}\right), 3.98-4.11(2 \mathrm{H}, \mathrm{m}, \mathrm{C}(2) \mathrm{H}$ and $\mathrm{C}(3) H), 5.79(1 \mathrm{H}$, $\mathrm{dd}, J 3.2,0.3, \operatorname{Ar} H), 6.08(1 \mathrm{H}, \mathrm{dd}, J 3.2,1.9, \operatorname{Ar} H), 7.11-7.14$ $(2 \mathrm{H}, \mathrm{m}, \mathrm{Ar} H), 7.20-7.24(4 \mathrm{H}, \mathrm{m}, \mathrm{ArH}) ; \delta_{\mathrm{C}}\left(125 \mathrm{MHz}, \mathrm{CDCl}_{3}\right)$ $37.4(C(3)), 38.5(C(4)), 52.5\left(C \mathrm{H}_{3}\right), 54.7(C(2)), 108.1(\operatorname{ArC})$, 110.3 ( $A r C), 115.5$ (q, $J$ 290, $\left.C F_{3}\right), 127.9$ (ArC), 128.3 (ArC), 128.7 ( $A r C), 136.1$ (C(2)ArC(1)), 141.7 (C(3)ArC(5)), 152.1 $(\mathrm{C}(3) \operatorname{ArC}(2)), 172.8(C(1)=\mathrm{O}), 189.2(\mathrm{q}, J 35.4, C(5)=\mathrm{O}) ; \mathrm{m} / \mathrm{z}$ $\left(\mathrm{NSI}^{+}\right) 341\left([\mathrm{M}+\mathrm{H}]^{+}, 100 \%\right) ; \delta_{\mathrm{F}}\left(470 \mathrm{MHz}, \mathrm{CDCl}_{3}\right)-79.4$ $\left(\mathrm{CF}_{3}\right)$; HRMS $\left(\mathrm{NSI}^{+}\right) \quad \mathrm{C}_{17} \mathrm{H}_{16} \mathrm{~F}_{3} \mathrm{O}_{4}{ }^{+} \quad\left([\mathrm{M}+\mathrm{H}]^{+}\right)$requires 341.0995; found 341.1000 (+1.4 ppm).

\section{Preparation of products (Table 4)}


(3R,4R)-methyl 2-oxo-3,4-diphenyl-3,4-dihydro-2H-pyran6-carboxylate 21. Following general procedure $\mathrm{B}$, anhydride $\mathbf{1}$ (63.5 mg, $0.25 \mathrm{mmol})$ in $\mathrm{CH}_{2} \mathrm{Cl}_{2}(2 \mathrm{~mL})$, HBTM-2.1 $(2 S, 3 R)-3$ (3.09 mg, $0.01 \mathrm{mmol}, 5 \mathrm{~mol} \%)$, keto ester 42 (38.0 mg, 0.20 mmol) and DIPEA $(43.5 \mu \mathrm{L}, 0.25 \mathrm{mmol})$ for $16 \mathrm{~h}$ at $-78{ }^{\circ} \mathrm{C}$ gave crude lactone $(3 R, 4 R)-21(98: 2 \mathrm{dr})$. Chromatographic purification (eluent $\mathrm{Et}_{2} \mathrm{O}$ :petrol 30:70) gave lactone $(3 R, 4 R)-21$ $(>99: 1 \mathrm{dr})$ as a white solid $(47.8 \mathrm{mg}, 78 \%)$ with identical spectroscopic data as previously reported ${ }^{11 \mathrm{a}}[\alpha]_{\mathrm{D}}{ }^{20}-195.0$ (c $\left.0.2, \mathrm{CH}_{2} \mathrm{Cl}_{2}\right)$; $\left\{\right.$ lit. $^{11 \mathrm{a}}[\alpha]_{\mathrm{D}}{ }^{20}-179.0\left(c 0.5, \mathrm{CH}_{2} \mathrm{Cl}_{2}\right)$ for $\left.91 \% e e\right\}$; Chiral HPLC Chiralpak AD-H (40\% IPA:hexane, flow rate 1 $\left.\mathrm{mL} \min ^{-1}, 211 \mathrm{~nm}, 30{ }^{\circ} \mathrm{C}\right) \mathrm{t}_{\mathrm{R}}(3 R, 4 R): 11.1 \mathrm{~min}, \mathrm{t}_{\mathrm{R}}(3 S, 4 S): 17.8$ $\min ,>99 \%$ ee.

(3R,4R)-methyl 3-(4-chlorophenyl)-2-oxo-4-phenyl-3,4dihydro-2H-pyran-6-carboxylate 22. Following general procedure $\mathrm{B}$, anhydride $55(80.8 \mathrm{mg}, 0.25 \mathrm{mmol})$ in $\mathrm{CH}_{2} \mathrm{Cl}_{2}(2$ mL), НBTM-2.1 (2S,3R)-3 (3.09 mg, $0.01 \mathrm{mmol}, 5 \mathrm{~mol} \%)$, keto ester 42 (38.0 $\mathrm{mg}, 0.20 \mathrm{mmol})$ and DIPEA $(43.5 \mu \mathrm{L}, 0.25$ $\mathrm{mmol})$ for $16 \mathrm{~h}$ at $-78{ }^{\circ} \mathrm{C}$ gave crude lactone $(3 R, 4 R)-22(90: 10$ $\mathrm{dr})$. Chromatographic purification (eluent $\mathrm{Et}_{2} \mathrm{O}$ :petrol 30:70) gave lactone $(3 R, 4 R)-\mathbf{2 2}(>99: 1 \mathrm{dr})$ as a white solid $(53.3 \mathrm{mg}$, $79 \%)$; mp 138-140 ${ }^{\circ} \mathrm{C}$; $[\alpha]_{\mathrm{D}}{ }^{20}-193.0\left(c \quad 0.1, \mathrm{CH}_{2} \mathrm{Cl}_{2}\right)$; Chiral HPLC Chiralpak AD-H (40\% IPA:hexane, flow rate $1 \mathrm{~mL}$ $\left.\min ^{-1}, 211 \mathrm{~nm}, 30{ }^{\circ} \mathrm{C}\right) \mathrm{t}_{\mathrm{R}}(3 R, 4 R): 13.2 \mathrm{~min}, \mathrm{t}_{\mathrm{R}}(3 S, 4 S): 19.1 \mathrm{~min}$, $>99 \%$ ee; $v_{\max }$ (ATR) 3059, 3030, $2957(\mathrm{C}-\mathrm{H}), 1778(\mathrm{C}=\mathrm{O})$, $1746(\mathrm{C}=\mathrm{O}), 1663$; Data for major diastereoisomer: $\delta_{\mathrm{H}}(300$ $\left.\mathrm{MHz}, \mathrm{CDCl}_{3}\right) 3.78-3.82\left(4 \mathrm{H}, \mathrm{m}, \mathrm{C}(3) \mathrm{H}\right.$ and $\left.\mathrm{CH}_{3}\right), 3.94(1 \mathrm{H}, \mathrm{dd}$, $J$ 10.1, 3.4, C(4)H), $6.60(1 \mathrm{H}, \mathrm{d}, J 3.5, \mathrm{C}(5) H), 6.91-6.95(4 \mathrm{H}$, $\mathrm{m}, \mathrm{Ar} H), 7.15-7.21(5 \mathrm{H}, \mathrm{m}, \mathrm{ArH}) ; \delta_{\mathrm{C}}\left(75 \mathrm{MHz}, \mathrm{CDCl}_{3}\right) 45.4$ $(C(4)), 52.0(C(3)), 52.9\left(C_{3}\right), 118.2(C(5)), 127.6(A r C)$, 128.1 (ArC), 129.0 ( $A r C), 129.2$ (ArC), 129.9 (ArC), 133.7 (4ry $A r C$ ), 133.9 (4ry $A r C$ ), 138.8 (4ry $A r C$ ), 142.0 (C(6)), 160.6 $\left(\mathrm{CO}_{2} \mathrm{CH}_{3}\right), 166.6(\mathrm{C}(2)) ; \mathrm{m} / \mathrm{z}\left(\mathrm{NSI}^{+}\right) 365\left([\mathrm{M}+\mathrm{Na}]^{+}, 70 \%\right)$; HRMS $\left(\mathrm{NSI}^{+}\right) \mathrm{C}_{19} \mathrm{H}_{15}{ }^{35} \mathrm{ClO}_{4}{ }^{+}\left([\mathrm{M}+\mathrm{Na}]^{+}\right)$requires 365.0551 ; found 365.0547 (-1.1 ppm).

(3R,4R)-methyl 2-oxo-4-phenyl-3-(thiophen-3-yl)-3,4dihydro-2H-pyran-6-carboxylate 23. Following general procedure $\mathrm{B}$, anhydride $58(66.6 \mathrm{mg}, 0.25 \mathrm{mmol})$ in $\mathrm{CH}_{2} \mathrm{Cl}_{2}(2$ mL), НВTM-2.1 (2S,3R)-3 (3.09 mg, $0.01 \mathrm{mmol}, 5 \mathrm{~mol} \%)$, keto ester $42(38.0 \mathrm{mg}, 0.20 \mathrm{mmol})$ and DIPEA $(43.5 \mu \mathrm{L}, 0.25$ $\mathrm{mmol})$ for $16 \mathrm{~h}$ at $-78{ }^{\circ} \mathrm{C}$ gave crude lactone $(3 R, 4 R)-23(93: 7$ dr). Chromatographic purification (eluent $\mathrm{Et}_{2} \mathrm{O}$ :petrol 30:70) gave lactone $(3 R, 4 R)-\mathbf{2 3}(>99: 1 \mathrm{dr})$ as a white solid $(52.3 \mathrm{mg}$, $83 \%)$; mp $128-130{ }^{\circ} \mathrm{C}$; $[\alpha]_{\mathrm{D}}{ }^{20}-209.5$ (c $\left.0.2, \mathrm{CH}_{2} \mathrm{Cl}_{2}\right)$; Chiral HPLC Chiralpak AD-H (40\% IPA:hexane, flow rate $1 \mathrm{~mL}$ $\left.\min ^{-1}, 211 \mathrm{~nm}, 30{ }^{\circ} \mathrm{C}\right) \mathrm{t}_{\mathrm{R}}(3 R, 4 R): 11.1 \mathrm{~min}, \mathrm{t}_{\mathrm{R}}(3 S, 4 S): 19.4 \mathrm{~min}$, $>99 \% e e ; v_{\max }$ (ATR) 3105, 2951, $2924(\mathrm{C}-\mathrm{H}), 1767(\mathrm{C}=\mathrm{O})$, $1736(\mathrm{C}=\mathrm{O}), 1661$; Data for major diastereoisomer: $\delta_{\mathrm{H}}(500$ $\left.\mathrm{MHz}, \mathrm{CDCl}_{3}\right) 3.81\left(3 \mathrm{H}, \mathrm{s}, \mathrm{CH}_{3}\right), 3.97(1 \mathrm{H}, \mathrm{dd}, J$ 7.1, 4.5, $\mathrm{C}(4) H), 4.01(1 \mathrm{H}, \mathrm{d}, J 7.1, \mathrm{C}(3) H), 6.62(1 \mathrm{H}, \mathrm{d}, J 4.4, \mathrm{C}(5) H)$, 6.90-6.91 (2H, m, ArH), $7.01(2 \mathrm{H}, \mathrm{d}, J$ 6.8, $\operatorname{Ar} H), 7.19-7.25$ $(4 \mathrm{H}, \mathrm{m}, \mathrm{ArH}) ; \delta_{\mathrm{C}}\left(75 \mathrm{MHz}, \mathrm{CDCl}_{3}\right) 44.9(C(4)), 48.0$ (C(3)), $52.9\left(\mathrm{CH}_{3}\right), 117.2(C(5)), 123.4(\mathrm{ArC}), 126.6(\mathrm{ArC}), 126.8$ $(A r C), 127.4(A r C), 128.1(A r C), 129.2(A r C), 135.1$ (4ry $A r C)$, 139.0 (4ry $A r C$ ), 142.1 (C(6)), $160.7\left(\mathrm{CO}_{2} \mathrm{CH}_{3}\right), 166.2$ (C(2)); $m / z\left(\mathrm{NSI}^{+}\right) 332\left(\left[\mathrm{M}+\mathrm{NH}_{4}\right]^{+}, 61 \%\right) ; \mathrm{HRMS}\left(\mathrm{NSI}^{+}\right) \mathrm{C}_{17} \mathrm{H}_{18} \mathrm{NO}_{4} \mathrm{~S}^{+}$ $\left(\left[\mathrm{M}+\mathrm{NH}_{4}\right]^{+}\right)$requires 332.0951; found 332.0955 (+1.2 ppm).

$(3 R, 4 R)$-methyl 4-(4-nitrophenyl)-2-oxo-3-phenyl-3,4dihydro-2H-pyran-6-carboxylate 24. Following general procedure $\mathrm{B}$, anhydride $\mathbf{1}(63.5 \mathrm{mg}, 0.25 \mathrm{mmol})$ in $\mathrm{CH}_{2} \mathrm{Cl}_{2}(2$ $\mathrm{mL}$, НВTM-2.1 (2S,3R)-3 (3.09 $\mathrm{mg}, 0.01 \mathrm{mmol}, 5 \mathrm{~mol} \%)$, keto ester 43 (47.0 mg, $0.20 \mathrm{mmol})$ and DIPEA (43.5 $\mu \mathrm{L}, 0.25$ $\mathrm{mmol})$ for $16 \mathrm{~h}$ at $-78{ }^{\circ} \mathrm{C}$ gave crude lactone $(3 R, 4 R)-24(91: 9$ $\mathrm{dr})$. Chromatographic purification (eluent $\mathrm{Et}_{2} \mathrm{O}$ :petrol 40:60) gave lactone $(3 R, 4 R)-\mathbf{2 4}(93: 7 \mathrm{dr})$ as an off-white solid (60.6 $\mathrm{mg}, 86 \%$ ) with identical spectroscopic data as previously reported; ${ }^{11 a}$ Chiral HPLC Chiralcel OD-H (50\% IPA:hexane,

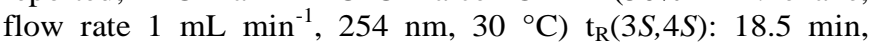
$\mathrm{t}_{\mathrm{R}}(3 R, 4 R): 23.2 \mathrm{~min},>99 \%$ ee.

$(3 R, 4 R)$-methyl 4-(4-methoxyphenyl)-2-oxo-3-phenyl-3,4dihydro-2H-pyran-6-carboxylate 25 . Following general procedure $\mathrm{B}$, anhydride $\mathbf{1}(63.5 \mathrm{mg}, 0.25 \mathrm{mmol})$ in $\mathrm{CH}_{2} \mathrm{Cl}_{2}(2$ $\mathrm{mL})$, НBTM-2.1 (2S,3R)-3 (3.09 $\mathrm{mg}, 0.01 \mathrm{mmol}, 5 \mathrm{~mol} \%)$, keto ester 44 (44.0 $\mathrm{mg}, 0.20 \mathrm{mmol})$ and DIPEA $(43.5 \mu \mathrm{L}, 0.25$ $\mathrm{mmol})$ for $16 \mathrm{~h}$ at $-78{ }^{\circ} \mathrm{C}$ gave crude lactone $(3 R, 4 R)-25(93: 7$ dr). Chromatographic purification (eluent $\mathrm{Et}_{2} \mathrm{O}$ :petrol 30:70) gave lactone $(3 R, 4 R)-\mathbf{2 5}(>99: 1 \mathrm{dr})$ as a white solid $(57.9 \mathrm{mg}$, $86 \%$ ) with identical spectroscopic data as previously reported; ${ }^{11 a}$ Chiral HPLC Chiralpak AD-H (40\% IPA:hexane, flow rate $\left.1 \mathrm{~mL} \mathrm{~min}^{-1}, 211 \mathrm{~nm}, 30{ }^{\circ} \mathrm{C}\right) \mathrm{t}_{\mathrm{R}}(3 R, 4 R)$ : $14.9 \mathrm{~min}$, $\mathrm{t}_{\mathrm{R}}(3 S, 4 S): 35.5 \mathrm{~min},>99 \%$ ee.

$(3 R, 4 R)$-methyl 4-(furan-2-yl)-2-oxo-3-phenyl-3,4-dihydro2H-pyran-6-carboxylate 26. Following general procedure $\mathrm{B}$, anhydride $1(63.5 \mathrm{mg}, 0.25 \mathrm{mmol})$ in $\mathrm{CH}_{2} \mathrm{Cl}_{2}(2 \mathrm{~mL})$, HBTM$2.1(2 S, 3 R)-3$ (3.09 $\mathrm{mg}, 0.01 \mathrm{mmol}, 5 \mathrm{~mol} \%)$, keto ester 45 (36.0 $\mathrm{mg}, 0.20 \mathrm{mmol})$ and DIPEA $(43.5 \mu \mathrm{L}, 0.25 \mathrm{mmol})$ for 16 $\mathrm{h}$ at $-78{ }^{\circ} \mathrm{C}$ gave crude lactone $(3 R, 4 R)-26(95: 5 \mathrm{dr})$. Chromatographic purification (eluent $\mathrm{Et}_{2} \mathrm{O}$ :petrol 30:70) gave lactone $(3 R, 4 R)-26(>99: 1 \mathrm{dr})$ as a white solid $(47.6 \mathrm{mg}, 80 \%)$ with identical spectroscopic data as previously reported; ${ }^{11 a}$ Chiral HPLC Chiralcel OJ-H (20\% IPA:hexane, flow rate $1 \mathrm{~mL}$ $\left.\min ^{-1}, 211 \mathrm{~nm}, 30{ }^{\circ} \mathrm{C}\right) \mathrm{t}_{\mathrm{R}}(3 R, 4 R): 25.9 \mathrm{~min}, \mathrm{t}_{\mathrm{R}}(3 S, 4 S): 29.1 \mathrm{~min}$, $>99 \%$ ee.

\section{Preparation of products (Table 5)}

(R)-methyl 2-(2-benzoyl-1-phenylhydrazinyl)-2phenylacetate 27 . Following general procedure $\mathrm{C}$, anhydride $\mathbf{1}$ (63.5 mg, $0.25 \mathrm{mmol})$ in $\mathrm{CH}_{2} \mathrm{Cl}_{2}(2 \mathrm{~mL}), \mathrm{HBTM}-2.1(2 S, 3 R)-3$ (3.09 $\mathrm{mg}, 0.01 \mathrm{mmol}, 5 \mathrm{~mol} \%)$, diazene 46 (42.0 $\mathrm{mg}, 0.20$ mmol) and DIPEA $(43.5 \mu \mathrm{L}, 0.25 \mathrm{mmol})$ for $30 \mathrm{mins}$ at $-78^{\circ} \mathrm{C}$, followed by addition of $\mathrm{MeOH}(2 \mathrm{~mL})$ and stirring for $1 \mathrm{~h}$ at $\mathrm{rt}$ gave, after chromatographic purification (eluent $\mathrm{Et}_{2} \mathrm{O}$ :petrol 50:50) a rotameric mixture (ratio 90:10) of hydrazide $(2 R)-27$ as a pale yellow solid (43.9 $\mathrm{mg}, 61 \%$ ) with identical spectroscopic data as previously reported; ${ }^{11 \mathrm{c}}[\alpha]_{\mathrm{D}}{ }^{20}-37.2(c 0.5$, $\left.\mathrm{CH}_{2} \mathrm{Cl}_{2}\right)$; $\left\{\right.$ lit. ${ }^{11 \mathrm{c}}[\alpha]_{\mathrm{D}}{ }^{20}-37.6\left(c 0.5, \mathrm{CH}_{2} \mathrm{Cl}_{2}\right)$ for $\left.99 \% e e\right\}$; Chiral HPLC Chiralpak IB (10\% IPA:hexane, flow rate $1 \mathrm{~mL}$ $\left.\min ^{-1}, 220 \mathrm{~nm}, 30^{\circ} \mathrm{C}\right) \mathrm{t}_{\mathrm{R}}(2 S): 10.5 \mathrm{~min}, \mathrm{t}_{\mathrm{R}}(2 R): 12.2 \mathrm{~min}, 98 \%$ $e e$.

(R)-methyl 2-(2-benzoyl-1-phenylhydrazinyl)-2-(4methoxyphenyl)acetate 28. Following general procedure $\mathrm{C}$, anhydride $56(78.5 \mathrm{mg}, 0.25 \mathrm{mmol})$ in $\mathrm{CH}_{2} \mathrm{Cl}_{2}(2 \mathrm{~mL})$, HBTM$2.1(2 S, 3 R)-3$ (3.09 mg, $0.01 \mathrm{mmol}, 5 \mathrm{~mol} \%)$, diazene 46 (42.0 $\mathrm{mg}, 0.20 \mathrm{mmol})$ and DIPEA $(43.5 \mu \mathrm{L}, 0.25 \mathrm{mmol})$ for $30 \mathrm{mins}$ at $-78{ }^{\circ} \mathrm{C}$, followed by addition of $\mathrm{MeOH}(2 \mathrm{~mL})$ and stirring for $1 \mathrm{~h}$ at $\mathrm{rt}$ gave, after chromatographic purification (eluent $\mathrm{Et}_{2} \mathrm{O}$ :petrol 40:60) a rotameric mixture (ratio 89:11) of hydrazide (2R)-28 as an off-white solid $(65.4 \mathrm{mg}, 84 \%)$ with identical spectroscopic data as previously reported; ${ }^{11 \mathrm{c}}$ Chiral HPLC Chiralpak IB (10\% IPA:hexane, flow rate $1 \mathrm{~mL} \mathrm{~min}^{-1}$, $\left.254 \mathrm{~nm}, 30{ }^{\circ} \mathrm{C}\right) \mathrm{t}_{\mathrm{R}}(2 S): 14.7 \mathrm{~min}, \mathrm{t}_{\mathrm{R}}(2 R): 19.6 \mathrm{~min}, 99 \%$ ee. 
(R)-methyl 2-(2-benzoyl-1-phenylhydrazinyl)-2-(4chlorophenyl)acetate 29. Following general procedure $\mathrm{C}$, anhydride $55(80.8 \mathrm{mg}, 0.25 \mathrm{mmol})$ in $\mathrm{CH}_{2} \mathrm{Cl}_{2}(2 \mathrm{~mL})$, HBTM2.1 (2S,3R)-3 (3.09 mg, $0.01 \mathrm{mmol}, 5 \mathrm{~mol} \%)$, diazene 46 (42.0 $\mathrm{mg}, 0.20 \mathrm{mmol})$ and DIPEA $(43.5 \mu \mathrm{L}, 0.25 \mathrm{mmol})$ for $30 \mathrm{mins}$ at $-78{ }^{\circ} \mathrm{C}$, followed by addition of $\mathrm{MeOH}(2 \mathrm{~mL})$ and stirring for $1 \mathrm{~h}$ at $\mathrm{rt}$ gave, after chromatographic purification (eluent $\mathrm{Et}_{2} \mathrm{O}$ :petrol 40:60) a rotameric mixture (ratio 91:9) of hydrazide (2R)-29 as a yellow solid (59.0 mg, 75\%); mp $135-137{ }^{\circ} \mathrm{C}$; $[\alpha]_{\mathrm{D}}{ }^{20}-12.4\left(c 0.5, \mathrm{CH}_{2} \mathrm{Cl}_{2}\right)$; Chiral HPLC Chiralpak IB $(10 \%$

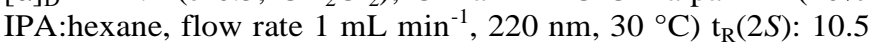
$\min , \mathrm{t}_{\mathrm{R}}(2 R)$ : $14.7 \mathrm{~min}, 97 \% e e ; v_{\max }(\mathrm{ATR}) 3341(\mathrm{~N}-\mathrm{H}), 3065$, $3028(\mathrm{C}-\mathrm{H}), 2949(\mathrm{C}-\mathrm{H}), 1726(\mathrm{C}=\mathrm{O}), 1686(\mathrm{C}=\mathrm{O})$; Data for major rotamer: $\delta_{\mathrm{H}}\left(300 \mathrm{MHz}, \mathrm{CDCl}_{3}\right) 3.72(3 \mathrm{H}, \mathrm{s}, \mathrm{CH}), 5.75$ $(1 \mathrm{H}, \mathrm{s}, \mathrm{C}(2) H), 6.87-6.93(3 \mathrm{H}, \mathrm{m}, \mathrm{Ar} H), 7.17-7.25(5 \mathrm{H}, \mathrm{m}$, $\mathrm{ArH}), 7.28-7.35$ (4H, m, ArH), 7.48-7.51 (2H, m, ArH), 8.50 $(1 \mathrm{H}, \mathrm{s}, \mathrm{NH}) ; \delta_{\mathrm{C}}\left(75 \mathrm{MHz}, \mathrm{CDCl}_{3}\right) 52.7\left(\mathrm{OCH}_{3}\right), 66.1(C(2))$, $114.9(\mathrm{~N} A r C(2,6)), 121.9(\mathrm{~N} A r C(4)), 127.0(\operatorname{ArC}), 128.7(\operatorname{ArC})$, 128.9 ( $A r C$ ), 129.5 (ArC), 130.5 (ArC), 131.9 (4ry ArC), 132.0 $(A r C), 132.7$ (4ry $A r C$ ), 134.9 (4ry $A r C$ ), 148.2 (4ry $A r C$ ), 166.6 $(\mathrm{NHC}=\mathrm{O}), 172.7(\mathrm{MeOC}=\mathrm{O})$; Selected data for minor rotamer: $\delta_{\mathrm{H}}\left(300 \mathrm{MHz}, \mathrm{CDCl}_{3}\right) 3.62\left(3 \mathrm{H}, \mathrm{s}, \mathrm{CH}_{3}\right), 5.46(1 \mathrm{H}, \mathrm{s}$, $\mathrm{C}(2) H), 6.79(2 \mathrm{H}, \mathrm{d}, J$ 8.4, $\mathrm{Ar} H), 7.88(1 \mathrm{H}, \mathrm{s}, \mathrm{N} H) ; \delta_{\mathrm{C}}(75$ $\left.\mathrm{MHz}, \mathrm{CDCl}_{3}\right) 52.2\left(\mathrm{OCH}_{3}\right), 67.2(C(2)), 115.3(\mathrm{~N} A r C(2,6))$, 122.5 (NArC(4)), 127.9 (ArC), $130.0(A r C), 131.4(A r C) ; \mathrm{m} / z$ $\left(\mathrm{NSI}^{+}\right) 395\left([\mathrm{M}+\mathrm{H}]^{+}, 100 \%\right)$; HRMS $\left(\mathrm{NSI}^{+}\right) \mathrm{C}_{22} \mathrm{H}_{20}{ }^{35} \mathrm{ClN}_{2} \mathrm{O}_{3}{ }^{+}$ $\left([\mathrm{M}+\mathrm{H}]^{+}\right)$requires 395.1157 ; found $395.1160(+0.8 \mathrm{ppm})$.

(R)-methyl 2-(2-benzoyl-1-phenylhydrazinyl)-2-(thiophen-3yl)acetate 30. Following general procedure $\mathrm{C}$, anhydride $\mathbf{5 8}$ (66.5 mg, $0.25 \mathrm{mmol})$ in $\mathrm{CH}_{2} \mathrm{Cl}_{2}(2 \mathrm{~mL}), \mathrm{HBTM}-2.1(2 S, 3 R)-3$ (3.09 $\mathrm{mg}, 0.01 \mathrm{mmol}, 5 \mathrm{~mol} \%$ ), diazene 46 (42.0 mg, 0.20 mmol) and DIPEA (43.5 $\mu \mathrm{L}, 0.25 \mathrm{mmol})$ for $30 \mathrm{mins}$ at $-78^{\circ} \mathrm{C}$, followed by addition of $\mathrm{MeOH}(2 \mathrm{~mL})$ and stirring for $1 \mathrm{~h}$ at $\mathrm{rt}$ gave, after chromatographic purification (eluent $\mathrm{Et}_{2} \mathrm{O}$ :petrol 40:60) a rotameric mixture (ratio 93:7) of hydrazide $(2 R)-\mathbf{3 0}$ as a yellow solid $(54.8 \mathrm{mg}, 75 \%)$ with identical spectroscopic data as previously reported; ${ }^{11 \mathrm{c}}$ Chiral HPLC Chiralpak IA (40\%

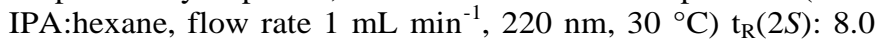
$\min , \mathrm{t}_{\mathrm{R}}(2 R): 22.2 \mathrm{~min}, 95 \%$ ee.

(R)-methyl 2-(2-(4-nitrobenzoyl)-1-phenylhydrazinyl)-2-
phenylacetate 31 . Following general procedure C, anhydride 1
$(63.5 \mathrm{mg}, 0.25 \mathrm{mmol})$ in $\mathrm{CH}_{2} \mathrm{Cl}_{2}(2 \mathrm{~mL})$, HBTM- $2.1(2 S, 3 R)-3$
$(3.09 \mathrm{mg}, 0.01 \mathrm{mmol}, 5 \mathrm{~mol} \%)$, diazene $48(51.0 \mathrm{mg}, 0.20$
mmol) and DIPEA $(43.5 \mu \mathrm{L}, 0.25 \mathrm{mmol})$ for $30 \mathrm{mins}$ at $-78{ }^{\circ} \mathrm{C}$,
followed by addition of MeOH $(2 \mathrm{~mL})$ and stirring for $1 \mathrm{~h}$ at $\mathrm{rt}$
gave, after chromatographic purification (eluent $\mathrm{Et}_{2} \mathrm{O}$ :petrol
$50: 50)$ a rotameric mixture (ratio $72: 28)$ of hydrazide $(2 R)-31$
as a pale yellow solid $(63.5 \mathrm{mg}$, $78 \%)$ with identical
spectroscopic data as previously reported; ${ }^{11 \mathrm{c}} \mathrm{Chiral} \mathrm{HPLC}$
Chiralcel OJ-H $\left(30 \%\right.$ IPA:hexane, flow rate $1 \mathrm{~mL} \mathrm{~min}^{-1}, 211$
nm, $\left.40{ }^{\circ} \mathrm{C}\right) \mathrm{t}_{\mathrm{R}}(2 S): 18.5 \mathrm{~min}, \mathrm{t}_{\mathrm{R}}(2 R): 14.0 \mathrm{~min}, 99 \% e e$.

(R)-methyl 2-(2-(4-methoxybenzoyl)-1-phenylhydrazinyl)-2phenylacetate 32. Following general procedure $\mathrm{C}$, anhydride 1 (63.5 mg, $0.25 \mathrm{mmol})$ in $\mathrm{CH}_{2} \mathrm{Cl}_{2}(2 \mathrm{~mL}), \mathrm{HBTM}-2.1(2 S, 3 R)-3$ (3.09 mg, $0.01 \mathrm{mmol}, 5 \mathrm{~mol} \%$ ), diazene 50 (48.0 mg, 0.20 mmol) and DIPEA $(43.5 \mu \mathrm{L}, 0.25 \mathrm{mmol})$ for $30 \mathrm{mins}$ at $-78^{\circ} \mathrm{C}$, followed by addition of $\mathrm{MeOH}(2 \mathrm{~mL})$ and stirring for $1 \mathrm{~h}$ at $\mathrm{rt}$ gave, after chromatographic purification (eluent $\mathrm{Et}_{2} \mathrm{O}$ :petrol 80:20) a rotameric mixture (ratio 91:9) of hydrazide (2R)-32 as a white solid $(60.7 \mathrm{mg}, 78 \%)$; mp $149-151{ }^{\circ} \mathrm{C} ;[\alpha]_{\mathrm{D}}{ }^{20}-21.6(c$ $0.5, \mathrm{CH}_{2} \mathrm{Cl}_{2}$ ); Chiral HPLC Chiralpak IB (10\% IPA:hexane, flow rate $\left.1 \mathrm{~mL} \min ^{-1}, 211 \mathrm{~nm}, 30{ }^{\circ} \mathrm{C}\right) \mathrm{t}_{\mathrm{R}}(2 S): 17.2 \mathrm{~min}, \mathrm{t}_{\mathrm{R}}(2 R)$ : $20.6 \mathrm{~min}, 99 \% e e ; v_{\max }(\mathrm{ATR}) 3281(\mathrm{~N}-\mathrm{H}), 2951,2932(\mathrm{C}-\mathrm{H})$, $1732(\mathrm{C}=\mathrm{O}), 1655(\mathrm{C}=\mathrm{O}), 1605(\mathrm{C}-\mathrm{O})$; Data for major rotamer: $\delta_{\mathrm{H}}\left(300 \mathrm{MHz}, \mathrm{CDCl}_{3}\right) 3.74\left(6 \mathrm{H}, \mathrm{s}, 2 \mathrm{CH}_{3}\right), 5.81(1 \mathrm{H}, \mathrm{s}, \mathrm{C}(2) H)$, 6.76-6.79 (2H, m, C $(\mathrm{O}) \operatorname{Ar}(3,5) H), 6.91-6.95(3 \mathrm{H}, \mathrm{m}, \operatorname{Ar} H)$, 7.18-7.25 (5H, m, ArH), 7.37-7.40 (2H, m, ArH), 7.44-7.47 $(2 \mathrm{H}, \mathrm{m}, \mathrm{C}(\mathrm{O}) \operatorname{Ar}(2,6) H), 8.37(1 \mathrm{H}, \mathrm{s}, \mathrm{NH}) ; \delta_{\mathrm{C}}\left(75 \mathrm{MHz}, \mathrm{CDCl}_{3}\right)$ $52.5\left(\mathrm{OCH}_{3}\right), 55.4\left(\mathrm{OCH}_{3}\right), 66.7(C(2)), 113.8(\mathrm{~N} A r C(3,5))$, 114.8 ( $A r C), 121.6(\mathrm{~N} A r C(4)), 125.0$ (4ry $A r C), 128.6(A r C)$, 128.9 (ArC), 128.9 (ArC), 129.1 (ArC), 129.5 (ArC), 133.3 (4ry $A r C), 148.5$ (4ry $A r C$ ), 162.4 (4ry $A r C$ ), $166.1(\mathrm{NHC}=\mathrm{O}), 173.1$ $(\mathrm{MeOC}=\mathrm{O})$; Selected data for minor rotamer: $\delta_{\mathrm{H}}(300 \mathrm{MHz}$, $\left.\mathrm{CDCl}_{3}\right) 3.63\left(3 \mathrm{H}, \mathrm{s}, \mathrm{CH}_{3}\right), 3.67\left(3 \mathrm{H}, \mathrm{s}, \mathrm{CH}_{3}\right), 5.54(1 \mathrm{H}, \mathrm{s}$, $\mathrm{C}(2) H), 6.48(2 \mathrm{H}, \mathrm{d}, J 8.9, \operatorname{Ar} H), 7.79(1 \mathrm{H}, \mathrm{s}, \mathrm{NH}) ; \delta_{\mathrm{C}}(75$ $\left.\mathrm{MHz}, \mathrm{CDCl}_{3}\right) 55.3\left(\mathrm{OCH}_{3}\right), 115.3(\mathrm{NArC}) ; \mathrm{m} / \mathrm{z}\left(\mathrm{NSI}^{+}\right) 391$ $\left([\mathrm{M}+\mathrm{H}]^{+}, \quad 100 \%\right) ; \quad \mathrm{HRMS} \quad\left(\mathrm{NSI}^{+}\right) \quad \mathrm{C}_{23} \mathrm{H}_{23} \mathrm{~N}_{2} \mathrm{O}_{4}{ }^{+} \quad\left([\mathrm{M}+\mathrm{H}]^{+}\right)$ requires 391.1652; found $391.1656(+0.9 \mathrm{ppm})$.

(R)-methyl 2-(2-(4-chlorobenzoyl)-1-phenylhydrazinyl)-2phenylacetate 33 . Following general procedure $\mathrm{C}$, anhydride $\mathbf{1}$ (63.5 mg, $0.25 \mathrm{mmol})$ in $\mathrm{CH}_{2} \mathrm{Cl}_{2}(2 \mathrm{~mL}), \mathrm{HBTM}-2.1(2 S, 3 R)-3$ (3.09 $\mathrm{mg}, 0.01 \mathrm{mmol}, 5 \mathrm{~mol} \%$ ), diazene 47 (48.9 $\mathrm{mg}, 0.20$ mmol) and DIPEA $(43.5 \mu \mathrm{L}, 0.25 \mathrm{mmol})$ for $30 \mathrm{mins}$ at $-78^{\circ} \mathrm{C}$, followed by addition of $\mathrm{MeOH}(2 \mathrm{~mL})$ and stirring for $1 \mathrm{~h}$ at $\mathrm{rt}$ gave, after chromatographic purification (eluent $\mathrm{Et}_{2} \mathrm{O}$ :petrol 40:60) a rotameric mixture (ratio 83:17) of hydrazide $(2 R)-33$ as a white solid $(58.1 \mathrm{mg}, 74 \%) ; \mathrm{mp} 63-66^{\circ} \mathrm{C} ;[\alpha]_{\mathrm{D}}{ }^{20}-30.8(c$ 0.5, $\mathrm{CH}_{2} \mathrm{Cl}_{2}$ ); Chiral HPLC Chiralpak IB (10\% IPA:hexane, flow rate $\left.1 \mathrm{~mL} \mathrm{~min}^{-1}, 220 \mathrm{~nm}, 30{ }^{\circ} \mathrm{C}\right) \mathrm{t}_{\mathrm{R}}(2 S): 10.9 \mathrm{~min}, \mathrm{t}_{\mathrm{R}}(2 R)$ : $12.2 \mathrm{~min}, 98 \%$ ee; $v_{\max }(\mathrm{ATR}) 3280(\mathrm{~N}-\mathrm{H}), 3032,2951(\mathrm{C}-\mathrm{H})$, $1732(\mathrm{C}=\mathrm{O}), 1663(\mathrm{C}=\mathrm{O}), 1597(\mathrm{C}-\mathrm{O})$; Data for major rotamer: $\delta_{\mathrm{H}}\left(500 \mathrm{MHz}, \mathrm{CDCl}_{3}\right) 3.84\left(3 \mathrm{H}, \mathrm{s}, \mathrm{CH}_{3}\right), 5.91(1 \mathrm{H}, \mathrm{s}, \mathrm{C}(2) H)$, 7.01-7.05 (3H, m, ArH), 7.32- $7.37(7 \mathrm{H}, \mathrm{m}, \mathrm{ArH}), 7.46-7.50$ $(4 \mathrm{H}, \mathrm{m}, \mathrm{Ar} H), 8.56(1 \mathrm{H}$, br s, $\mathrm{N} H) ; \delta_{\mathrm{C}}\left(75 \mathrm{MHz}, \mathrm{CDCl}_{3}\right) 52.6$ $\left(\mathrm{OCH}_{3}\right), 66.7(C(2)), 114.8(\mathrm{~N} A r C(2,6)), 121.9(\mathrm{~N} A r C(4))$, 128.5 (ArC), 128.7 (ArC), 128.9 (ArC), $129.0(A r C), 129.1$ $(A r C), 129.5$ ( $A r C$ ), 131.2 (4ry $A r C$ ), 133.2 (4ry $A r C$ ), 138.1 (4ry $A r C$ ), 148.3 (4ry $A r C$ ), $165.6 \quad(\mathrm{NHC}=\mathrm{O}), 173.1$ $(\mathrm{MeOC}=\mathrm{O})$; Selected data for minor rotamer: $\delta_{\mathrm{H}}(500 \mathrm{MHz}$, $\left.\mathrm{CDCl}_{3}\right) 3.72\left(3 \mathrm{H}, \mathrm{s}, \mathrm{CH}_{3}\right), 5.61(1 \mathrm{H}, \mathrm{s}, \mathrm{C}(2) H), 6.90(2 \mathrm{H}, \mathrm{d}, J$ 8.5, $\mathrm{ArH}), 7.13(1 \mathrm{H}, \mathrm{t}, J$ 7.3, $\mathrm{ArH}), 7.21(2 \mathrm{H}, \mathrm{t}, J$ 7.7, $\mathrm{ArH})$, $7.26(2 \mathrm{H}, \mathrm{d}, J 8.1, \mathrm{ArH}), 8.05(1 \mathrm{H}$, br s, $\mathrm{NH}) ; \delta_{\mathrm{C}}(75 \mathrm{MHz}$, $\left.\mathrm{CDCl}_{3}\right) 52.5\left(\mathrm{OCH}_{3}\right), 68.1(C(2)), 115.5(\mathrm{~N} A r C(2,6)), 122.6$ $(\mathrm{N} A r C(4)), 127.4(A r C), 129.3(A r C), 130.0$ (ArC), 130.2 $(A r C) ; \quad m / z \quad\left(\mathrm{NSI}^{+}\right) 395\left([\mathrm{M}+\mathrm{H}]^{+}, 100 \%\right) ; \mathrm{HRMS}\left(\mathrm{NSI}^{+}\right)$ $\mathrm{C}_{22} \mathrm{H}_{20}{ }^{35} \mathrm{ClN}_{2} \mathrm{O}_{3}{ }^{+}\left([\mathrm{M}+\mathrm{H}]^{+}\right)$requires 395.1157 ; found 395.1159 (+0.5 ppm).

(R)-methyl 2-(2-(4-methylbenzoyl)-1-phenylhydrazinyl)-2phenylacetate 34 . Following general procedure $\mathrm{C}$, anhydride 1 (63.5 mg, $0.25 \mathrm{mmol})$ in $\mathrm{CH}_{2} \mathrm{Cl}_{2}(2 \mathrm{~mL}), \mathrm{HBTM}-2.1(2 S, 3 R)-3$ (3.09 $\mathrm{mg}, 0.01 \mathrm{mmol}, 5 \mathrm{~mol} \%$ ), diazene 49 (44.8 $\mathrm{mg}, 0.20$ mmol) and DIPEA $(43.5 \mu \mathrm{L}, 0.25 \mathrm{mmol})$ for $30 \mathrm{mins}$ at $-78^{\circ} \mathrm{C}$, followed by addition of $\mathrm{MeOH}(2 \mathrm{~mL})$ and stirring for $1 \mathrm{~h}$ at $\mathrm{rt}$ gave, after chromatographic purification (eluent $\mathrm{Et}_{2} \mathrm{O}$ :petrol 50:50) a rotameric mixture (ratio 92:8) of hydrazide $(2 R)-34$ as a white solid $(63.2 \mathrm{mg}, 85 \%)$ with identical spectroscopic data as previously reported; ${ }^{11 \mathrm{c}}$ Chiral HPLC Chiralpak IB $(10 \%$ IPA:hexane, flow rate $\left.1 \mathrm{~mL} \min ^{-1}, 220 \mathrm{~nm}, 30^{\circ} \mathrm{C}\right) \mathrm{t}_{\mathrm{R}}(2 S): 11.7$ $\min , \mathrm{t}_{\mathrm{R}}(2 R): 13.5 \mathrm{~min}, 99 \%$ ee.

\section{Acknowledgements}

We thank the Royal Society for a University Research Fellowship (A.D.S), the Carnegie Trust for the Universities of 
Scotland (L.C.M) and EPSRC (A.D.H) for funding. C.F and the research leading to these results has received funding from the European Research Council under the European Union's Seventh Framework Programme (FP7/2007-2013) / ERC grant agreement $\mathrm{n}^{\circ}$ 279850. We also thank the EPSRC National Mass Spectrometry Service Centre (Swansea).

\section{Notes and references}

${ }^{a}$ EaStCHEM, School of Chemistry, University of St Andrews, North Haugh, St Andrews, KY16 9ST, UK.

Electronic Supplementary Information (ESI) available: $\left[{ }^{1} \mathrm{H}\right.$ and ${ }^{13} \mathrm{C}$ NMR spectra of all novel compounds and relevant HPLC traces]. See DOI: $10.1039 / \mathrm{b} 000000 \mathrm{x} /$

${ }^{1}$ For a comprehensive review of Lewis base catalysis see S. E. Denmark and G. L. Beutner, Angew. Chem., Int. Ed., 2008, 47, 1560.

For a review of ammonium enolate chemistry see M. J. Gaunt and C. C. C. Johansson, Chem. Rev., 2007, 107, 5596.

For general reviews on organocatalysis see: (a) A. Dondoni and A. Massi, Angew. Chem. Int. Ed., 2008, 47, 4638; (b) D. W. C. MacMillan, Nature, 2008, 455, 304.

For a review of the chemistry and reactivity of enolates derived from ketenes see D. H. Paull, A. Weatherwax and T. Lectka, Tetrahedron, 2009, 65, 6771. For select recent examples see: (a) J. M. Berlin and G. C. Fu, Angew. Chem., Int. Ed., 2008, 47, 7048; (b) M. Dochnahl and G. C. Fu, Angew. Chem., Int. Ed., 2009, 48, 2391; (c) D. H. Paull, M. T. Scerba, E. Alden-Danforth, L. R. Widger and T. Lectka, J. Am. Chem. Soc., 2008, 123, 17260.

${ }^{5}$ For select examples see: (a) B. List, Acc. Chem. Res., 2004, 37, 548; (b) W. Notz, F. Tanaka and C. F. Barbas, Acc. Chem. Res., 2004, 37, 580; (c) Y. Chi and S. H. Gellman, J. Am. Chem. Soc., 2006, 128, 6804; (d) J. Yang, C. Chandler, M. Stadler, D. Kampen and B. List, Nature, 2008, 452, 453.

For reviews see: (a) D. Enders and T. Balensiefer, Acc. Chem. Res., 2004, 37, 534; (b) N. Marion, S. Diez-Gonzalez and S. P. Nolan, Angew. Chem. Int. Ed., 2007, 46, 2988. (c) J. Douglas, G. Churchill and A. D. Smith, Synthesis, 2012, 134, 20421.

For reviews see: (a) T. Marcelli and H. Hiemstra, Synthesis, 2010, 1229; (b) T. Ooi and K. Maruoka, Angew. Chem. Int. Ed., 2007, 46, 4222.

For select examples of Romo's pioneering work regarding intramolecular NCAL reactions see: (a) G. S. Cortez, R. L. Tennyson and D. Romo, J. Am. Chem. Soc., 2001, 123, 7945; (b) H. Henry-Riyad, C. Lee. V. C. Purohit and D. Romo, Org. Lett., 2006, 8, 4363; (c) V. C. Purohit, A. S. Matla and D. Romo, J. Am. Chem. Soc., 2008, 130, 10478; (d) C. Leverett, V. C. Purohit and D. Romo, Angew. Chem. Int. Ed., 2010, 49, 9479; (e) G. Liu and D. Romo, Angew. Chem. Int. Ed., 2011, 50, 7537.

For a recent review detailing the use of isothioureas in nucleophilic catalysis see J. E. Taylor, S. D. Bull and J. M. J. Williams, Chem. Soc. Rev., 2012, 14, 2109. For kinetic resolutions using anhydrides as acylating agents see: (a) V. B. Birman and X. Li, Org. Lett., 2006, 8, 1351; (b) Q. Xu, H. Zhou, X. Geng and P. Chen, Tetrahedron, 2009, 65, 2232; (c) D. Belmessieri, C. Joannesse, P. A. Woods, C. MacGregor, C. Jones, C. D. Campbell, C. P. Johnston, N. Duguet, C. Concellón, R. A. Bragg and A. D. Smith, Org. Biomol. Chem., 2011, 9, 559. For determination of absolute configuration using isothioureas in kinetic resolutions see (d) A. J. Wagner, J. G. David and S. D. Rychnovsky, Org. Lett., 2011, 13, 4470. For kinetic resolutions using carboxylic acids as acylating agents utilising in situ formation of a reactive mixed anhydride see: (e) I. Shiina and K. Nakata, Tetrahedron Lett., 2007, 48, 8314; (f) I. Shiina, K. Nakata, K. Ono, Y-S. Onda and M. Itagaki, J. Am. Chem. Soc., 2010, 132, 11629. For the kinetic resolution of $\beta$-lactams see (g) V. D. Bumbu and V. B. Birman, J. Am. Chem. Soc., 2011, 133, 13902. For desymmetrisation processes see: (h) X. Yang, G. Lu and V. B. Birman, Org. Lett., 2010, 12, 892; (i) X. Yang and V. B. Birman, Angew. Chem. Int. Ed., 2011, 50, 5553. For asymmetric $C$-acylation and $C$-carboxylation reaction processes see: (j) C. Joannesse, C. P. Johnston, C. Concellón, C. Simal, D. Philp and A. D. Smith Angew. Chem., Int. Ed., 2009, 48, 8914; (k) B. Viswambharan, T. Okimura, S. Suzuki and S. Okamoto, J. Org. Chem., 2011, 76, 6678; (1) P. A. Woods, L. C. Morrill, R. A. Bragg and A. D. Smith, Chem. Eur. J., 2011, 17, 11060.

For pioneering work see: (a) V. B. Birman, H. Jiang, X. Li, L. Guo and E. W. Uffman, J. Am. Chem. Soc., 2006, 128, 6536; (b) M. Kobayashi and S. Okamoto, Tetrahedron Lett., 2006, 47, 4347.

For examples see: (a) D. Belmessieri, L. C. Morrill, C. Simal, A. M. Z. Slawin and A. D. Smith. J. Am. Chem. Soc., 2011, 133, 2714; (b) C. Simal, T.
Lebl, A. M. Z. Slawin and A. D. Smith, Angew. Chem. Int. Ed., 2012, 51, 3653; (c) L. C. Morrill, T. Lebl, A. M. Z. Slawin and A. D. Smith, Chem. Sci., 2012, 3, 2088; (d) D. Belmessieri, D. B. Cordes, A. M. Z. Slawin and A. D. Smith, Org. Lett., 2013, 15, 3472; (e) L. C. Morrill, J. Douglas, T. Lebl, A. M. Z. Slawin, D. J. Fox and A. D. Smith, Chem. Sci., 2013, 4, 4146.

For selected other examples of Lewis base catalyzed reaction processes from this laboratory see: (a) J. E. Thomson, K. Rix and A. D. Smith, Org. Lett., 2006, 8, 3785; (b) N. Duguet, C. D. Campbell, A. M. Z. Slawin and A. D. Smith, Org. Biomol. Chem., 2008, 6, 1108; (c) C. Concellón, N. Duguet and A. D. Smith, Adv. Synth. Catal., 2009, 351, 3001; (d) P. A. Woods, L. C. Morrill, R. A. Bragg and A. D. Smith, Org. Lett., 2010, 12, 2660; (e) K. B. Ling and A. D. Smith, Chem. Commun., 2011, 47, 373.

E. R. T. Robinson, C. Fallan, C. Simal, A. M. Z. Slawin and A. D. Smith, Chem. Sci., 2013, 4, 2193.

(a) C. Cornaggia, F. Manoni, E. Torrente, S. Tallon and S. J. Connon, Org. Lett., 2012, 14, 1850; (b) F. Manoni, C. Cornaggia, J. Murray, S. Tallon and S. J. Connon, Chem. Commun., 2012, 48, 6502.

(a) L. Hao, Y. Du, H. Lv, X. Chen, H. Jiang, Y. Shao and Y. R. Chi, Org. Lett., 2012, 14, 2154; (b) L. Hao, C. W. Chuen, R. Ganguly and Y. R. Chi, Synlett, 2013, 24, 1197; (c) J. Cheng, Z. Huang and Y. R. Chi, Angew. Chem. Int. Ed., 2013, 52, 1; (d) Z. Fu, J. Xu, T. Zhu, W. W. Y. Leong and Y. R. Chi, Nature Chem., DOI. 10.1038/nchem.1710.

${ }^{16}$ See experimental section for further details regarding the synthesis of anhydrides.

${ }_{17}$ At the moment this process is limited to the use of 2-arylacetic anhydrides with other anhydrides (such as propionic anhydride) giving less than $5 \%$ product conversions. We postulate that mnay be possibly due to preferential Claisen-type self condensation of the anhydride and current investigations are directed towards optimisation of these reaction processes.

${ }_{18}^{18}$ J. A. Jenkins and T. Cohen, J. Org. Chem., 1975, 40, 3566.

${ }^{19}$ A. Jonathan and M. J. Layva, WO2010/17408 A1, 2010.

${ }^{20}$ A. A. M. Roof, H. F. van Woerden and H. Cerfontain, Tetrahedron, 1976, 32, 2967.

${ }_{22}^{21}$ No literature melting point quoted.

${ }^{22}$ W. J. L. Wood, A. W. Patterson, H. J. Hirojuki, K. Rishi and J. A. Ellman, J. Am. Chem. Soc., 2005, 127, 15521. 